Article

\title{
The Effects of Air Pollution on Firms' Internal Control Quality: Evidence from China
}

\author{
Siyi Liu, Daoguang Yang *, Nian Liu and Xin Liu \\ Department of Accounting, University of International Business and Economics, Beijing 100029, China; \\ liusiyi@uibe.edu.cn (S.L.); rpg163@126.com (N.L.); xinliu@uibe.edu.cn (X.L.) \\ * Correspondence: dgyang@uibe.edu.cn
}

Received: 13 August 2019; Accepted: 13 September 2019; Published: 17 September 2019

\begin{abstract}
Research on the consequences of air pollution has tended to focus on the macro and environmental effects on human health, often ignoring micro effects. In this paper, we empirically investigate the micro-institutional costs of air pollution, and our results show that in China, firms' internal control quality is significantly and negatively associated with the severity of air pollution in its home city and that the most significant effects of air pollutants are those of PM2.5 and $\mathrm{SO}_{2}$, confirming that air pollution incurs micro-institutional costs. We find that this effect varies depending on factors related to the environment, ownership structure, the demographic traits of the board of directors' chairman, and employees. Further analysis indicates that air pollution can degrade the quality of accounting information, provoke agency problems, and lower firm value. This study reveals the micro-institutional costs of air pollution and identifies the mechanisms by which air pollution affects the quality of macroeconomic development. By so doing, this study enables China's government and public to better its understanding of air pollution and recognize the value of the Blue-Sky Protection Campaign. This study also reinforces the importance of the transition toward a new economic mode based on "high-quality development," which will play a vital role in China's new era.
\end{abstract}

Keywords: air pollution; internal control; costs; institution

JEL Classification: G31; Q53; D22

\section{Introduction}

The report of the 19th National Congress of the Communist Party of China (CPC) recognizes that the country is progressing from a high-speed growth stage to a high-quality development stage, and this shift has profound implications for economic development strategies. The rapid growth of the last several decades, however, has taken its toll, with air pollution representing one of the heaviest costs. Empirical evidence indicates that air pollution has become one of the main factors damaging China's public image and impeding its high-quality economic development [1]. Therefore, the General Secretary of CPC Central Committee, Xi Jinping, has proposed to "persistently implement air pollution prevention and governance actions to win the battle against the Blue-Sky Protection Campaign" in the 19th CPC National Congress. These systematic efforts require the joint participation of all stakeholders, including the government, the market, and micro-individuals [2]. However, progress could be slowed without a comprehensive understanding of air pollution and its hazards. Currently, the public approaches air pollution from the perspective of human health, both physical and mental (e.g., [3-6]), without thinking much about the extent to which air pollution may incur economic and social costs (e.g., [7]). Although several recent studies empirically examine air pollution in terms of economic and social considerations (e.g., [8-13]), these studies have two limitations and 
thus speak to the need for more research. First, the studies in question analyze readily apparent and short-term costs (effects on personal consumption and firm productivity), while ignoring the institutional costs that have the potential to be more serious and far-reaching. China's transition to a new mode of economic development, described above, entails a related transition from "extensive input" to one on "intensive management" and, correspondingly, from "resource dividend" to "institutional dividend." Second, studies tend to focus on either individual health or economic and social issues, barely touching the interlinking subject of firms. Even more importantly, as one of the major producers of air pollution, self-interested firms and their investors can hardly be expected to advance the concept of green development if they fail to recognize that air pollution harms their own interests.

Motivated to address this gap in the literature, this paper empirically tests the impact of air pollution on a firm's internal control quality using city-level air pollution data from China and a sample of non-financial public firms listed on the Shanghai and Shenzhen stock exchanges from 2014 to 2016. Of all possible institutional infrastructure and arrangements, we choose internal control for the following reasons: (1) theoretically, internal control is the most comprehensive and systematic institutional system, covering all aspects of firm operations and management [14,15]; and (2) in reality, high-quality internal control systems play a positive role in improving a firm's information environment (e.g., [16]), reducing financing costs (e.g., [17-19]), improving investment and operation efficiency (e.g., [20,21]), and increasing firm value (e.g., [22]). Our results show that a firm's internal control quality is significantly and negatively associated with the severity of air pollution in its home city and that among the effects of all measured air pollutants, those of PM2.5 and $\mathrm{SO}_{2}$ are the most significant, thus confirming that air pollution incurs micro-institutional costs. We find that this effect varies depending on factors related to the environment, ownership structure, the demographic traits of the board of directors' chairman, and employees. Further analysis indicates that air pollution can degrade accounting information quality, provoke agency problems, and lower firm value.

This paper contributes to the literature in the following ways. First, we are the first to investigate the micro-institutional costs of air pollution from the perspective of internal control, given that related research focuses on human physical and mental health (e.g., [3,4,10]). Studies addressing social and economic costs do not explore the costs to micro-institutions, the latter of which can be more serious.

Second, this paper extends the literature on the factors influencing a firm's internal control quality by finding evidence that air pollution degrades its quality.

Typically, studies have pursued this question by considering a firm's fundamental features (e.g., [16,17]) and corporate governance (e.g., [23-27]). Recent studies have stimulated interest in how regional cultural traditions may influence a firm's internal control quality (e.g., [28]). Nevertheless, studies have yet to explore the extent to which the ecological or natural environment can influence a firm's internal control quality. This paper fills this gap with its focus on air pollution.

Third, this study identifies a mechanism that links the two formerly separate research topics of micro-level human health, on the one hand, and macro-level social and economic issues, on the other hand. In this effort, this study moves beyond the dichotomy that is currently visible in research on air pollution (e.g., $[8,9,11,13])$. Finally, this study's results and conclusions can help raise the awareness of firms and stakeholders and thereby strengthen their commitment to a green economy characterized by high-quality development.

The rest of the paper is organized as follows. Section 2 reviews the literature and develops research hypotheses. Section 3 introduces the study's data, variables, and model. Section 4 reports the results of the main tests and offers analysis. Section 5 includes the robustness tests. Finally, Section 6 offers a summary and concluding remarks. 


\section{Literature Review and Hypothesis Development}

\subsection{Literature on the Consequences of Air Pollution}

Early studies on the consequences of air pollution examine its effects on human physical and mental health, and one branch of the literature links air pollution not only to various respiratory and cardiopulmonary diseases $[3,29]$ but also to death $[4,30]$. Supporting these findings, a World Health Organization survey shows that about 3 million people around the world die from air pollution each year and 1 in every 8-9 deaths result from air pollution [31,32]. Also, besides the obvious consequences of air pollution, there are less visible but still serious ones, such as exposure to pollutants resulting in general and physical fatigue [33], anxiety [5], depression [6], and even suicide [34].

In recent years, research attention has begun to turn to the areas of individual behavior and economic and social costs. For example, Chang et al. [10] investigate how air pollution affects individual decisions when purchasing insurance in China and find that the number of contracts increases by $7.2 \%$ for each standard deviation increase in air pollution; Cheung and Law [35] show that $30 \%$ of international tourists, when considering destinations, perceive of air pollution as a concern. Another group of scholars analyze the effects of air pollution on work input and/or output, reaching mutually consistent conclusions. Chang et al. [8] find that the decrease of outdoor PM2.5 significantly reduces workers' packaging speed, and estimate that the decrease of PM2.5 in the US from 1999 to 2008 saved 19.5 billion dollars in labor costs. Using a natural experiment offered by smelter closures in Mexico City, Hanna and Oliva [11] show that the drop of one unit of $\mathrm{SO}_{2}$ leads to an increase of 1.3 in work hours per week, with workers less likely to be sick. Zivin and Neidell [13] and Chang et al. [6] find that air pollution (ozone and PM2.5) significantly decreases the working efficiency of American agricultural workers and hotline telephone operators, respectively.

In China, empirical evidence confirms that haze significantly decreased the country's economic development from 2004 to 2013. Unlike the above research on the economic costs of air pollution, Herrnstadt et al. [36] and Lu et al. [7] explore its social costs. Herrnstadt et al. [36] find that air pollution in Chicago and Los Angeles significantly increases violent crime (such as personal assaults). Using annual data on 9360 cities in the US and experimental survey data, Lu et al. [7] verify their prediction that air pollution increases the probability of both violent crimes (including murder, rape, robbery, attack and theft) and the tendency to cheat.

\subsection{Literature on the Determinants of Internal Control Quality}

The literature on internal control quality approaches the question of its determinants and characteristics from two angles.

First, a firm's internal control quality is closely related to its most basic traits. Doyle et al. [16] demonstrate that internal control material weaknesses (ICMWs) are negatively associated with firm size, age, and financial conditions, and positively associated with firm growth, operation complexity, and restructuring. Ashbaugh-Skaife et al. [17] find similar results for 538 firms with internal control weaknesses between November 2002 and December 2005. Finally, research on non-profit organizations indicates that ICMWs are more likely to emerge in organizations that are financially weak, growing, more complex, and smaller (e.g., [37]). In China, some scholars produce consistent results when using information derived from the voluntary disclosures of internal control attestations as measures of measure internal control quality.

Second, a firm's internal controls are affected by both its internal governance and its external environment. The first factor speaks to the nature and structure of ownership. For example, Gong et al. [38] find that firms whose managers control their firms and have voting rights in excess of their cash flow rights are more likely to misreport internal control weaknesses. Second, the governance of the audit committee is another consideration. Krishnan [26] shows that firms with more audit committee members with independence and/or financial expertise are less likely to have internal control problems. Zhang et al. [1], Goh [24], Hoitash et al. [39], and Johnstone et al. [25] produce 
consistent findings regarding the remediation of ICMWs. Third, management is an influencing factor. Li et al. [40] show that firms receiving adverse auditors' opinions of their internal controls generally do not have competent CFOs and that the opinion improves after hiring a more qualified CFO. Balsam et al. [23] find that management equity incentives are significantly negatively correlated with the presence of ICMWs. Fourth, the external environment plays a role. Yun [41] finds that oversight of the legal system and governmental intervention significantly affect internal control quality, while the role of informal mechanisms such as the media is not significant. Recently, some scholars have started to explore the effect of regional cultural traditions on firms' internal control quality. A study conducted by Kanagaretnam et al. [28] shows that national culture (i.e., individualism, power distance, and uncertainty avoidance) is an important factor determining the effectiveness of firms' internal control.

\subsection{Hypotheses Development}

The quality (or effectiveness) of internal control reflects two indispensable dimensions: the degree to which an internal control system has been both fully established and effectively implemented. Hence, internal control quality is affected by how a firm's internal governance and external environment affect these two dimensions. As an important external ecological factor, air pollution can influence internal control quality in the following ways, as research suggests.

In the absence of a complete internal control system, air pollution can have a negative effect on a firm and result in a management that is less motivated, less effective, and less able to construct an internal control system. That task is complex and requires a systematic approach that calls upon concentration, resilience, and endurance. Those requirements notwithstanding, people with considerable exposure to air pollution can show symptoms of anxiety [5,7], depression [6,34], and fatigue [33]. In clinical practice, anxiety is generally manifested as a feeling of apprehension or fear, and commonly entails restlessness, insecurity, and distraction; depression can manifest as people's doubting themselves, being pessimistic, losing interest in daily life, and showing signs of fatigue. In such a psychological state, management avoids difficult tasks, not to mention the complex and "tricky" task of constructing an internal control system. Even if management is willing or obliged or to do so, their efficiency and effectiveness may be diminished.

Air pollution can also be expected to harm the implementation of an internal control system. Medical studies have shown that an uncomfortable air environment affects human physiological functions (such as serum levels and male hormones) and may result in some becoming extremely aggressive (e.g., [42,43]). This physical disequilibrium can emerge in behavior that is often regarded as fearless and defiant, and that challenges the prevailing rules and constraints. Consistent with their findings, Lu et al. [7] and Herrnstadt et al. [36] find that air pollution increases the incidence of violent crime and other problematic behavior. Therefore, the findings above support the prediction that a firm's employees (including internal shareholders, management, and staff, etc.) exposed to severe air pollution are expected to show a certain degree of disregard for the internal control system designed to constrain and discipline activity in the decision-making and execution domain, suggesting that the implementation of that firm's internal control system is ineffective.

Collectively, we propose the following hypothesis:

H1. Ceteris paribus, a firm's internal control quality is negatively associated with the severity of air pollution in its home city.

The opposite situation may also exist. As business involves trade-offs between risks and benefits, it is anticipated that firms are likely to seek formal and institutional responses to the adverse effects of air pollution (e.g., [44]). In addition, the anxiety and depression aggravated by air pollution are reflected in a sense of insecurity, and thus a high-quality internal control system may be adopted to reduce the uncertainty facing management and shareholders. Therefore, we propose the following hypothesis, one that is countervailing to $\mathrm{H} 1$ : 
H2. Ceteris paribus, internal control quality is positively associated with the severity of air pollution in cities where the firm is located.

\section{Research Design}

\subsection{Data and Sample Selection}

Our initial sample consists of Chinese A-share public firms listed in the Shanghai and Shenzhen stock exchanges from 2014 to 2016. After eliminating the financial industry and observations with missing data on air pollution or other relevant variables, we obtain a sample of 7211 firm-year observations involving 627 city-years. We use four types of data. The first type is air pollution data retrieved from the Chinese Research Data Service (CNRDS) database. The CNRDS includes daily air pollution (quality) data for all cities in China since 2013. As only 60 days' data were collected for each city in 2013, we exclude that year from our sample. The daily air pollution (quality) index is calculated by the 24-h average density of sulfur dioxide $\left(\mathrm{SO}_{2}\right)$, nitrogen dioxide $\left(\mathrm{NO}_{2}\right)$, carbon monoxide $(\mathrm{CO})$, ozone $\left(\mathrm{O}_{3}\right), \mathrm{PM} 2.5$, and PM10. The higher the index, the worse the air pollution. The second type of data concerns internal control quality, which is found in Chen et al.'s [45] Internal Control Index of Chinese listed firms. This index is designed based on the Basic Standard for Enterprise Internal Control (hereafter, Standard) and its corresponding guidelines (hereafter, Guidelines), the COSO's (1992) [46] integrated framework for internal control, and the guidelines, rules, and related regulations issued by the Shanghai and Shenzhen Stock Exchange. Third, this study uses macro data that include the annual GDP data of all cities obtained from the China Entrepreneur Investment Club database, and the data speaking to the regional degree of marketization and market product development are obtained from Wang et al. (2016) [47]. Fourth, data on other variables are retrieved from CSMAR database. To avoid the influence of outliers, all the continuous variables are winsorized at $1 \%$ and $99 \%$.

\subsection{Model Construction}

Following the literature [16], our model specification is as follows:

$$
I C_{i, t}=\alpha_{0}+\alpha_{1} A P_{c, i, t}+\sum_{i=2}^{16} \alpha_{i} X_{i}+\text { Industry \& Year }+\varepsilon_{i, t}
$$

where IC represents internal control quality, measured as the standardized score of that year's Internal Control Index. In the robustness test, we define IC in another way. The air pollution (AP) proxy for the degree of air pollution in the home cities of firms is calculated as the average score of the Air Pollution Index over the year divided by 100 . Here, $X$ represents four types of control variables included in the model: (1) the firm's basic features, including firm size (SIZE), return on assets (ROA), financial leverage $(L E V)$, equity refinancing $(S E O)$, growth $(G R O W)$, and inventory intensity $(I N V E)$; (2) the firms' operational complexity, e.g., mergers and acquisition $(M A)$, the number of business segments $(S E G)$, foreign sales (EXPO), and years since listing $(A G E)$; (3) corporate governance, including the size of the board of directors (BORD), the duality of the chairman and CEO (DUAL), the shares held by the largest shareholder (TOP1), whether is it audited by a "Big Four" audit firm (BIG4), and the type of the firm's ultimate owner (OWNER); (4) macro-level variables, including annual economic status (GDP) and the degree of marketization in the firm's home province (MKT). Please see Table 1 for the specific definitions of the variables. In addition, the model controls for industry and year fixed effects. If $\alpha_{1}$ is significantly negative, then $\mathrm{H} 1$ is supported, and if $\alpha_{1}$ is significantly positive, then $\mathrm{H} 2$ is verified. 
Table 1. Variables Definition.

\begin{tabular}{|c|c|}
\hline Variables & Definitions \\
\hline IC & $\begin{array}{l}\text { Internal control quality, measured as the standardized score of the Internal Control Index } \\
\text { of the year. }\end{array}$ \\
\hline$A P$ & $\begin{array}{l}\text { Air pollution, measured as the average of daily air pollution index over on year (from } 1 \text { January } \\
\text { to } 31 \text { December) in the city where the firm is located, and then divided by } 100 \text {. }\end{array}$ \\
\hline SIZE & Firm size, calculated as the natural logarithm of total asset at year end (in million CNY). \\
\hline ROA & Return on assets, calculated as pretax income divided by total assets at year end. \\
\hline$L E V$ & Financial leverage, calculated as the total liabilities divided by the total assets. \\
\hline SEO & $\begin{array}{l}\text { Equity refinancing, calculated as cash flows through equity financing divided by the total assets } \\
\text { at year end. }\end{array}$ \\
\hline GROW & $\begin{array}{l}\text { Growth, calculated as changes in sales from the last year to current years divided by sales in of } \\
\text { the last year. }\end{array}$ \\
\hline INVE & Inventory intensity, calculated as net inventory at year end divided by total assets at year end. \\
\hline$M A$ & $\begin{array}{l}\text { Mergers and acquisitions (M\&A), an indicator that equals } 1 \text { if M\&A occurs in the year, } \\
\text { and } 0 \text { otherwise. }\end{array}$ \\
\hline SEGM & $\begin{array}{l}\text { Business segments, calculated as the natural logarithm of one plus the number of business } \\
\text { segments. }\end{array}$ \\
\hline EXPO & Foreign sales, an indicator that equals 1 if foreign sales occur during the year, and 0 otherwise. \\
\hline AGE & Listing years, calculated as the logarithm of 1 plus the number of years the firm has been listed. \\
\hline BORD & The size of board of directors, calculated as the logarithm of the number of board directors. \\
\hline DUAL & Duality, an indicator that equals 1 if the chairman is also the CEO, and 0 otherwise. \\
\hline TOP1 & $\begin{array}{l}\text { The shares hold by the largest shareholder, calculated as the number of shares the largest } \\
\text { shareholder holds divided by the number of total shares. }\end{array}$ \\
\hline BIG4 & $\begin{array}{l}\text { "Big Four" auditors, an indicator if the firm is audited by a "Big Four" audit firm, } \\
\text { and } 0 \text { otherwise. }\end{array}$ \\
\hline OWNER & $\begin{array}{l}\text { The types of ultimate owners, an indicator that equals } 0 \text { for firms with no controller, } 1 \text { for private } \\
\text { companies, } 2 \text { to } 5 \text {, respectively, for firms controlled by the county, city, province, and the central } \\
\text { government. }\end{array}$ \\
\hline GDP & $\begin{array}{l}\text { The macroeconomic condition, measured as the GDP growth rate of the city where the firm is } \\
\text { located in. }\end{array}$ \\
\hline MKT & $\begin{array}{l}\text { The degree of marketization, measured as the Marketization Index ranking of the province } \\
\text { where the listed firm is in year } t \text { (equaling } 0 \text { to } 2 \text {, respectively, from low to high ranking). }\end{array}$ \\
\hline
\end{tabular}

\section{Empirical Results and Analysis}

\subsection{Descriptive Statistics}

Table 2 presents the descriptive statistics of the main variables. The mean value of IC is 0.510 , and the standard deviation is 0.283 , indicating significant variation in the internal control quality over time. The mean and median values of $A P$ are around 0.80 , and the standard deviation is 0.227 . The third quartile of $A P$ is 1.003 , which means that at least $25 \%$ of firm-years are exposed to polluted air. In addition, the descriptive statistical results of the other control variables show that each variable has variances in different firm-years.

\subsection{Multivariate Regression Analysis}

Table 3 presents the results of the main test of air pollution and the firm's internal control quality. Column (1) shows the result after controlling for a firms' basic features. The coefficient of $A P$ is -0.040 and is significant at the $5 \%$ level, indicating that internal control quality is lower when air pollution is more serious. In addition, SIZE and INVE are significantly positively correlated, while $L E V, S E O$, and GROW are significantly negatively correlated with $I C$, suggesting that firms with larger sizes and higher inventory intensity has higher internal control quality, while firms with higher financial leverage, higher amount of equity financing, and higher growth have lower internal control quality, which are, in general, in line with our expectations. In column (2), we further control for corporate operational characteristics. The coefficient of AP drops to -0.032 , but is still significant at the $10 \%$ level. The coefficient on SEGM and AGE are significantly positive, consistent with findings. Furthermore, 
in column (3), we include variables related to corporate governance. The coefficient of $A P$ is -0.045 and remains significant at a level of 5\%. In column (4), we include local GDP growth rate (GDP) and Marketization Index (MKT) in the model to mitigate concerns over omitting variables because the degree of air pollution in a region is often closely related to local economic development and institutional environment. AP remains negative at the $5 \%$ significance level with a coefficient of -0.041 . Overall, the results show that air pollution leads to the deterioration of the firm's internal control, and thus H1 is supported.

Table 2. Descriptive Statistics.

\begin{tabular}{ccccccc}
\hline Variables & Obs. & Mean & Std. Dev. & $\mathbf{2 5 \%}$ & Median & $\mathbf{7 5 \%}$ \\
\hline IC & 7227 & 0.510 & 0.283 & 0.268 & 0.515 & 0.756 \\
AP & 7227 & 0.824 & 0.227 & 0.674 & 0.804 & 1.003 \\
SIZE & 7227 & 8.379 & 1.265 & 7.500 & 8.226 & 9.088 \\
ROA & 7227 & 0.049 & 0.055 & 0.024 & 0.046 & 0.076 \\
LEV & 7227 & 0.432 & 0.210 & 0.263 & 0.418 & 0.591 \\
SEO & 7227 & 0.039 & 0.086 & 0.000 & 0.000 & 0.020 \\
GROW & 7227 & 0.191 & 0.571 & -0.054 & 0.081 & 0.258 \\
INVE & 7227 & 0.147 & 0.145 & 0.056 & 0.109 & 0.181 \\
MA & 7227 & 0.307 & 0.461 & 0.000 & 0.000 & 1.000 \\
SEGM & 7227 & 2.410 & 0.940 & 1.792 & 2.398 & 2.996 \\
EXPO & 7227 & 0.284 & 0.451 & 0.000 & 0.000 & 1.000 \\
AGE & 7227 & 2.215 & 0.737 & 1.609 & 2.303 & 2.890 \\
BORD & 7227 & 2.128 & 0.199 & 1.946 & 2.197 & 2.197 \\
DUAL & 7227 & 0.261 & 0.439 & 0.000 & 0.000 & 1.000 \\
TOP1 & 7227 & 0.344 & 0.148 & 0.226 & 0.325 & 0.446 \\
BIG4 & 7227 & 0.055 & 0.227 & 0.000 & 0.000 & 0.000 \\
OWNER & 7227 & 2.102 & 1.561 & 1.000 & 1.000 & 4.000 \\
GDP & 7227 & 0.078 & 0.036 & 0.067 & 0.083 & 0.099 \\
MKT & 7227 & 1.653 & 0.615 & 1.000 & 2.000 & 2.000 \\
\hline
\end{tabular}

Table 3. Air Pollution and Internal Control Quality.

\begin{tabular}{|c|c|c|c|c|}
\hline & (1) & (2) & (3) & (4) \\
\hline Dep. Variable $=$ & IC & IC & IC & IC \\
\hline$A P$ & $\begin{array}{c}-0.040 * * \\
(-2.15)\end{array}$ & $\begin{array}{c}-0.032 * \\
(-1.72)\end{array}$ & $\begin{array}{c}-0.045 * * \\
(-2.38)\end{array}$ & $\begin{array}{c}-0.041 * * \\
(-2.16)\end{array}$ \\
\hline SIZE & $\begin{array}{c}0.092 * * * \\
(22.73)\end{array}$ & $\begin{array}{c}0.082 \text { *** } \\
(16.77)\end{array}$ & $\begin{array}{c}0.071^{* * *} \\
(13.38)\end{array}$ & $\begin{array}{c}0.072^{* * *} \\
(13.54)\end{array}$ \\
\hline$R O A$ & $\begin{array}{l}-0.045 \\
(-0.58)\end{array}$ & $\begin{array}{l}0.008 \\
(0.10)\end{array}$ & $\begin{array}{l}0.021 \\
(0.27)\end{array}$ & $\begin{array}{l}-0.002 \\
(-0.03)\end{array}$ \\
\hline$L E V$ & $\begin{array}{c}-0.173^{* * *} \\
(-6.69)\end{array}$ & $\begin{array}{c}-0.193^{* * *} \\
(-7.45)\end{array}$ & $\begin{array}{c}-0.194^{* * *} \\
(-7.52)\end{array}$ & $\begin{array}{c}-0.187^{* * *} \\
(-7.23)\end{array}$ \\
\hline$S E O$ & $\begin{array}{c}-0.136^{* * *} \\
(-3.60)\end{array}$ & $\begin{array}{c}-0.103^{* * *} \\
(-2.73)\end{array}$ & $\begin{array}{c}-0.084^{* *} \\
(-2.21)\end{array}$ & $\begin{array}{c}-0.081^{* *} \\
(-2.14)\end{array}$ \\
\hline GROW & $\begin{array}{c}-0.043^{* * *} \\
(-7.23)\end{array}$ & $\begin{array}{c}-0.043^{* * *} \\
(-7.19)\end{array}$ & $\begin{array}{c}-0.039 * * * \\
(-6.48)\end{array}$ & $\begin{array}{c}-0.039 * * * \\
(-6.47)\end{array}$ \\
\hline INVE & $\begin{array}{c}0.088^{* *} \\
(2.38)\end{array}$ & $\begin{array}{c}0.086^{* *} \\
(2.29)\end{array}$ & $\begin{array}{c}0.083^{* *} \\
(2.21)\end{array}$ & $\begin{array}{c}0.077^{* *} \\
(2.06)\end{array}$ \\
\hline$M A$ & & $\begin{array}{l}-0.009 \\
(-1.25)\end{array}$ & $\begin{array}{l}-0.004 \\
(-0.49)\end{array}$ & $\begin{array}{l}-0.003 \\
(-0.45)\end{array}$ \\
\hline SEGM & & $\begin{array}{c}0.015^{* * * *} \\
(2.81)\end{array}$ & $\begin{array}{c}0.020^{* * * *} \\
(3.62)\end{array}$ & $\begin{array}{c}0.018^{* * * *} \\
(3.24)\end{array}$ \\
\hline
\end{tabular}


Table 3. Cont.

\begin{tabular}{|c|c|c|c|c|}
\hline & (1) & (2) & (3) & (4) \\
\hline EXPO & & $\begin{array}{l}0.005 \\
(0.57)\end{array}$ & $\begin{array}{l}0.004 \\
(0.49)\end{array}$ & $\begin{array}{l}0.002 \\
(0.29)\end{array}$ \\
\hline$A G E$ & & $\begin{array}{c}0.033^{* * *} \\
(5.08)\end{array}$ & $\begin{array}{c}0.024^{* * *} \\
(3.50)\end{array}$ & $\begin{array}{c}0.027^{* * *} \\
(3.86)\end{array}$ \\
\hline$B O R D$ & & & $\begin{array}{l}0.033 \\
(1.56)\end{array}$ & $\begin{array}{c}0.033 \\
(1.59)\end{array}$ \\
\hline$D U A L$ & & & $\begin{array}{c}-0.018 \text { ** } \\
(-1.98)\end{array}$ & $\begin{array}{c}-0.019 * * \\
(-2.11)\end{array}$ \\
\hline TOP1 & & & $\begin{array}{l}0.023 \\
(0.78)\end{array}$ & $\begin{array}{l}0.021 \\
(0.71)\end{array}$ \\
\hline BIG4 & & & $\begin{array}{l}0.032 \\
(1.60)\end{array}$ & $\begin{array}{l}0.027 \\
(1.34)\end{array}$ \\
\hline OWNER & & & $\begin{array}{c}0.013^{* * *} \\
(3.99)\end{array}$ & $\begin{array}{c}0.014^{* * *} \\
(4.12)\end{array}$ \\
\hline$G D P$ & & & & $\begin{array}{l}0.071 \\
(0.65)\end{array}$ \\
\hline$M K T$ & & & & $\begin{array}{c}0.025^{* * *} \\
(3.43)\end{array}$ \\
\hline Intercept & $\begin{array}{c}-0.166^{* * *} \\
(-3.48)\end{array}$ & $\begin{array}{c}-0.190^{* * *} \\
(-3.89)\end{array}$ & $\begin{array}{c}-0.187^{* * *} \\
(-2.90)\end{array}$ & $\begin{array}{c}-0.234^{* * *} \\
(-3.59)\end{array}$ \\
\hline Year E Ind. & Yes & Yes & Yes & Yes \\
\hline$N$ & 7227 & 7227 & 7227 & 7227 \\
\hline Adj. $R^{2}$ & 0.131 & 0.138 & 0.144 & 0.147 \\
\hline
\end{tabular}

$*, * *$, and ${ }^{* * *}$ indicate statistical significance at the $10 \%, 5 \%$, and $1 \%$ levels, respectively. The $t$-values are shown in parentheses. The standard errors are clustered at the firm level.

\section{Further Analysis and Robustness Tests}

\subsection{Further Analysis on Specific Internal Controls and Air Pollutants}

\subsubsection{Analysis of Internal Control Components}

Table 4 provides evidence that air pollution influences two components of internal control, risk assessment and information and communication, which suggests that air pollution weakens the effectiveness of risk assessment and reduces the firm's willingness to provide transparent information or to facilitate communication. This finding confirms our hypothesis regarding firms: air pollution can lead to a firm's ignoring risk, exacerbating anxiety, doubt, fatigue, and other psychological conditions, and reducing the willingness and efficiency of communication. It should be noted that this result does not suggest that air pollution plays no role in the other three components of internal control. As the five components of internal control display a form of interrelated integration, rather than their simple accumulation, air pollution's effects may be reflected in any one of those components $[45,48]$.

\subsubsection{Analysis of Specific Air Pollutants}

Table 5 reports the effect of each pollutant in the API on internal control quality. We replace our main variable of interest, $A P$, with haze $(P M 2.5, P M 10)$, sulfur dioxide $\left(\mathrm{SO}_{2}\right)$, nitrogen dioxide $\left(\mathrm{NO}_{2}\right)$, carbon monoxide $(\mathrm{CO})$, and ozone $\left(\mathrm{O}_{3}\right)$, respectively, from columns (1) to (6). The results show that only the coefficients of $\mathrm{PM} 2.5$ and $\mathrm{SO}_{2}$ are significantly negative, indicating that among air pollutants, 
haze (PM2.5) and sulfur dioxide $\left(\mathrm{SO}_{2}\right)$ are the main pollutants that deteriorate the quality of internal control quality.

Table 4. Regression Results for the Five Components of Internal Control.

\begin{tabular}{|c|c|c|c|c|c|}
\hline & (1) & (2) & (3) & (4) & (5) \\
\hline Dep. Variable $=$ & $\begin{array}{c}\text { Internal } \\
\text { Environment }\end{array}$ & Risk Assessment & $\begin{array}{c}\text { Control } \\
\text { Activities }\end{array}$ & $\begin{array}{l}\text { Information and } \\
\text { Communication }\end{array}$ & Monitoring \\
\hline$A P$ & $\begin{array}{l}-0.015 \\
(-0.76)\end{array}$ & $\begin{array}{c}-0.036 * \\
(-1.93)\end{array}$ & $\begin{array}{l}0.007 \\
(0.39)\end{array}$ & $\begin{array}{c}-0.085^{* * *} \\
(-3.92)\end{array}$ & $\begin{array}{l}0.017 \\
(1.04)\end{array}$ \\
\hline SIZE & $\begin{array}{c}0.071^{* * *} \\
(13.95)\end{array}$ & $\begin{array}{c}0.038^{* * *} \\
(7.08)\end{array}$ & $\begin{array}{c}0.037 * * * \\
(7.05)\end{array}$ & $\begin{array}{c}0.049^{* * *} \\
(8.38)\end{array}$ & $\begin{array}{l}0.012 * * \\
(2.57)\end{array}$ \\
\hline$R O A$ & $\begin{array}{l}0.206^{* * *} \\
(2.63)\end{array}$ & $\begin{array}{l}-0.127^{*} \\
(-1.68)\end{array}$ & $\begin{array}{l}-0.002 \\
(-0.02)\end{array}$ & $\begin{array}{l}-0.014 \\
(-0.17)\end{array}$ & $\begin{array}{l}0.050 \\
(0.71)\end{array}$ \\
\hline$L E V$ & $\begin{array}{c}-0.172 * * * \\
(-6.80)\end{array}$ & $\begin{array}{c}-0.083^{* * *} \\
(-3.29)\end{array}$ & $\begin{array}{c}-0.162 * * * \\
(-6.34)\end{array}$ & $\begin{array}{c}-0.111^{* * *} \\
(-3.83)\end{array}$ & $\begin{array}{l}-0.007 \\
(-0.29)\end{array}$ \\
\hline SEO & $\begin{array}{c}-0.125^{* * *} \\
(-3.28)\end{array}$ & $\begin{array}{l}-0.012 \\
(-0.32)\end{array}$ & $\begin{array}{l}-0.067 \\
(-1.63)\end{array}$ & $\begin{array}{c}-0.120^{* * *} \\
(-2.97)\end{array}$ & $\begin{array}{l}0.042 \\
(1.00)\end{array}$ \\
\hline GROW & $\begin{array}{c}-0.029 * * * \\
(-4.69)\end{array}$ & $\begin{array}{c}-0.019^{* * *} \\
(-3.03)\end{array}$ & $\begin{array}{c}-0.026^{* * *} \\
(-3.96)\end{array}$ & $\begin{array}{c}-0.019^{* * *} \\
(-2.88)\end{array}$ & $\begin{array}{c}-0.025 * * * \\
(-3.79)\end{array}$ \\
\hline INVE & $\begin{array}{c}0.181 * * * \\
(4.81)\end{array}$ & $\begin{array}{l}-0.035 \\
(-0.90)\end{array}$ & $\begin{array}{c}0.104^{* *} \\
(2.58)\end{array}$ & $\begin{array}{l}-0.027 \\
(-0.67)\end{array}$ & $\begin{array}{l}0.047 \\
(1.39)\end{array}$ \\
\hline$M A$ & $\begin{array}{c}-0.019 * * * \\
(-2.62)\end{array}$ & $\begin{array}{l}0.013 * \\
(1.68)\end{array}$ & $\begin{array}{l}0.002 \\
(0.27)\end{array}$ & $\begin{array}{l}0.004 \\
(0.46)\end{array}$ & $\begin{array}{l}-0.007 \\
(-0.90)\end{array}$ \\
\hline SEGM & $\begin{array}{l}-0.000 \\
(-0.06)\end{array}$ & $\begin{array}{l}0.010 * \\
(1.86)\end{array}$ & $\begin{array}{l}0.008 \\
(1.51)\end{array}$ & $\begin{array}{c}0.025 * * * \\
(4.25)\end{array}$ & $\begin{array}{l}0.008^{*} \\
(1.69)\end{array}$ \\
\hline EXPO & $\begin{array}{l}-0.010 \\
(-1.11)\end{array}$ & $\begin{array}{c}-0.018^{* *} \\
(-1.99)\end{array}$ & $\begin{array}{l}-0.002 \\
(-0.27)\end{array}$ & $\begin{array}{l}0.012 \\
(1.26)\end{array}$ & $\begin{array}{c}0.024 * * * \\
(2.88)\end{array}$ \\
\hline$A G E$ & $\begin{array}{l}-0.012 * \\
(-1.85)\end{array}$ & $\begin{array}{c}0.034^{* * *} \\
(5.07)\end{array}$ & $\begin{array}{l}0.009 \\
(1.33)\end{array}$ & $\begin{array}{l}0.009 \\
(1.19)\end{array}$ & $\begin{array}{c}0.034^{* * *} \\
(5.76)\end{array}$ \\
\hline$B O R D$ & $\begin{array}{l}0.013 \\
(0.61)\end{array}$ & $\begin{array}{l}-0.006 \\
(-0.31)\end{array}$ & $\begin{array}{l}0.040 * \\
(1.81)\end{array}$ & $\begin{array}{l}0.019 \\
(0.80)\end{array}$ & $\begin{array}{l}-0.001 \\
(-0.04)\end{array}$ \\
\hline DUAL & $\begin{array}{l}-0.000 \\
(-0.03)\end{array}$ & $\begin{array}{l}-0.005 \\
(-0.62)\end{array}$ & $\begin{array}{c}-0.021 \text { ** } \\
(-2.18)\end{array}$ & $\begin{array}{l}-0.001 \\
(-0.09)\end{array}$ & $\begin{array}{c}-0.017 \text { ** } \\
(-2.09)\end{array}$ \\
\hline TOP1 & $\begin{array}{l}-0.012 \\
(-0.43)\end{array}$ & $\begin{array}{c}0.067^{* *} \\
(2.26)\end{array}$ & $\begin{array}{l}0.012 \\
(0.41)\end{array}$ & $\begin{array}{l}-0.004 \\
(-0.11)\end{array}$ & $\begin{array}{l}0.023 \\
(0.90)\end{array}$ \\
\hline BIG4 & $\begin{array}{c}0.096^{* * *} \\
(5.20)\end{array}$ & $\begin{array}{l}-0.014 \\
(-0.68)\end{array}$ & $\begin{array}{l}-0.013 \\
(-0.63)\end{array}$ & $\begin{array}{l}0.028 \\
(1.26)\end{array}$ & $\begin{array}{l}-0.006 \\
(-0.37)\end{array}$ \\
\hline OWNER & $\begin{array}{c}0.015^{* * *} \\
(4.32)\end{array}$ & $\begin{array}{l}0.007^{* *} \\
(2.17)\end{array}$ & $\begin{array}{l}0.008 \text { ** } \\
(2.36)\end{array}$ & $\begin{array}{l}-0.003 \\
(-0.90)\end{array}$ & $\begin{array}{c}0.008^{* * *} \\
(2.91)\end{array}$ \\
\hline GDP & $\begin{array}{l}0.016 \\
(0.15)\end{array}$ & $\begin{array}{l}-0.044 \\
(-0.40)\end{array}$ & $\begin{array}{l}0.056 \\
(0.53)\end{array}$ & $\begin{array}{l}-0.012 \\
(-0.10)\end{array}$ & $\begin{array}{l}0.092 \\
(0.92)\end{array}$ \\
\hline MKT & $\begin{array}{c}0.033^{* * *} \\
(4.37)\end{array}$ & $\begin{array}{c}0.022 * * * \\
(3.02)\end{array}$ & $\begin{array}{c}0.017^{* *} \\
(2.24)\end{array}$ & $\begin{array}{l}0.007 \\
(0.89)\end{array}$ & $\begin{array}{c}-0.020 * * * \\
(-3.17)\end{array}$ \\
\hline Intercept & $\begin{array}{l}-0.088 \\
(-1.37)\end{array}$ & $\begin{array}{l}0.078 \\
(1.17)\end{array}$ & $\begin{array}{l}0.080 \\
(1.17)\end{array}$ & $\begin{array}{l}0.097 \\
(1.33)\end{array}$ & $\begin{array}{c}0.272 * * * \\
(4.94)\end{array}$ \\
\hline Year E Ind. & Yes & Yes & Yes & Yes & Yes \\
\hline$N$ & 7227 & 7227 & 7227 & 7227 & 7227 \\
\hline Adj. $R^{2}$ & 0.129 & 0.048 & 0.041 & 0.071 & 0.033 \\
\hline
\end{tabular}

$*, * *$, and ${ }^{* * *}$ indicate statistical significance at the $10 \%, 5 \%$, and $1 \%$ levels, respectively. The $t$-values are shown in parentheses. The standard errors are clustered at the firm level.. 
Table 5. Air Pollutants and Internal Control Quality.

\begin{tabular}{|c|c|c|c|c|c|c|}
\hline & (1) & (2) & (3) & (4) & (5) & (6) \\
\hline Dep. Var. $=$ & IC & IC & IC & IC & IC & IC \\
\hline PM2.5 & $\begin{array}{c}-0.054 * * \\
(-2.23)\end{array}$ & & & & & \\
\hline PM10 & & $\begin{array}{l}-0.024 \\
(-1.61)\end{array}$ & & & & \\
\hline $\mathrm{SO}_{2}$ & & & $\begin{array}{c}-0.072 * * \\
(-2.27)\end{array}$ & & & \\
\hline $\mathrm{NO}_{2}$ & & & & $\begin{array}{l}-0.059 \\
(-1.35)\end{array}$ & & \\
\hline $\mathrm{CO}$ & & & & & $\begin{array}{l}0.002 \\
(0.15)\end{array}$ & \\
\hline $\mathrm{O}_{3}$ & & & & & & $\begin{array}{l}-0.059 \\
(-1.35)\end{array}$ \\
\hline SIZE & $\begin{array}{c}0.072^{* * *} \\
(13.54)\end{array}$ & $\begin{array}{c}0.071^{* * *} \\
(13.51)\end{array}$ & $\begin{array}{c}0.071^{* * *} \\
(13.55)\end{array}$ & $\begin{array}{c}0.071^{* * *} \\
(13.46)\end{array}$ & $\begin{array}{c}0.071^{* * *} \\
(13.38)\end{array}$ & $\begin{array}{c}0.071^{* * *} \\
(13.43)\end{array}$ \\
\hline$R O A$ & $\begin{array}{l}-0.002 \\
(-0.03)\end{array}$ & $\begin{array}{l}-0.002 \\
(-0.03)\end{array}$ & $\begin{array}{l}-0.004 \\
(-0.05)\end{array}$ & $\begin{array}{l}-0.001 \\
(-0.02)\end{array}$ & $\begin{array}{l}-0.004 \\
(-0.05)\end{array}$ & $\begin{array}{l}-0.003 \\
(-0.04)\end{array}$ \\
\hline$L E V$ & $\begin{array}{c}-0.187^{* * * *} \\
(-7.23)\end{array}$ & $\begin{array}{c}-0.187^{* * *} \\
(-7.20)\end{array}$ & $\begin{array}{c}-0.186^{* * *} \\
(-7.18)\end{array}$ & $\begin{array}{c}-0.187^{* * *} \\
(-7.22)\end{array}$ & $\begin{array}{c}-0.188^{* * *} \\
(-7.24)\end{array}$ & $\begin{array}{c}-0.189 * * * \\
(-7.27)\end{array}$ \\
\hline SEO & $\begin{array}{c}-0.082 * * \\
(-2.15)\end{array}$ & $\begin{array}{c}-0.081 * * \\
(-2.12)\end{array}$ & $\begin{array}{c}-0.081 * * \\
(-2.12)\end{array}$ & $\begin{array}{c}-0.081 * * \\
(-2.12)\end{array}$ & $\begin{array}{c}-0.079 * * \\
(-2.08)\end{array}$ & $\begin{array}{c}-0.079 * * \\
(-2.08)\end{array}$ \\
\hline GROW & $\begin{array}{c}-0.039 * * * \\
(-6.46)\end{array}$ & $\begin{array}{c}-0.039^{* * *} \\
(-6.49)\end{array}$ & $\begin{array}{c}-0.039 * * * \\
(-6.51)\end{array}$ & $\begin{array}{c}-0.039^{* * *} \\
(-6.48)\end{array}$ & $\begin{array}{c}-0.039^{* * *} \\
(-6.50)\end{array}$ & $\begin{array}{c}-0.039^{* * *} \\
(-6.50)\end{array}$ \\
\hline INVE & $\begin{array}{c}0.078^{* *} \\
(2.07)\end{array}$ & $\begin{array}{c}0.078^{* *} \\
(2.08)\end{array}$ & $\begin{array}{c}0.080 \text { ** } \\
(2.13)\end{array}$ & $\begin{array}{c}0.078^{* *} \\
(2.08)\end{array}$ & $\begin{array}{c}0.079^{* *} \\
(2.10)\end{array}$ & $\begin{array}{c}0.078^{* *} \\
(2.09)\end{array}$ \\
\hline$M A$ & $\begin{array}{l}-0.003 \\
(-0.45)\end{array}$ & $\begin{array}{l}-0.004 \\
(-0.48)\end{array}$ & $\begin{array}{l}-0.004 \\
(-0.48)\end{array}$ & $\begin{array}{l}-0.003 \\
(-0.46)\end{array}$ & $\begin{array}{l}-0.004 \\
(-0.50)\end{array}$ & $\begin{array}{l}-0.004 \\
(-0.50)\end{array}$ \\
\hline SEGM & $\begin{array}{c}0.018^{* * * *} \\
(3.25)\end{array}$ & $\begin{array}{c}0.018^{* * *} \\
(3.29)\end{array}$ & $\begin{array}{c}0.017^{* * * *} \\
(3.21)\end{array}$ & $\begin{array}{c}0.019^{* * * *} \\
(3.46)\end{array}$ & $\begin{array}{c}0.019^{* * * *} \\
(3.48)\end{array}$ & $\begin{array}{c}0.019^{* * *} \\
(3.49)\end{array}$ \\
\hline EXPO & $\begin{array}{l}0.002 \\
(0.29)\end{array}$ & $\begin{array}{l}0.002 \\
(0.28)\end{array}$ & $\begin{array}{l}0.002 \\
(0.28)\end{array}$ & $\begin{array}{l}0.002 \\
(0.24)\end{array}$ & $\begin{array}{l}0.002 \\
(0.24)\end{array}$ & $\begin{array}{l}0.002 \\
(0.28)\end{array}$ \\
\hline$A G E$ & $\begin{array}{c}0.026^{* * *} \\
(3.84)\end{array}$ & $\begin{array}{c}0.027^{* * *} \\
(3.94)\end{array}$ & $\begin{array}{c}0.028^{* * *} \\
(4.03)\end{array}$ & $\begin{array}{c}0.027^{* * *} \\
(3.91)\end{array}$ & $\begin{array}{c}0.028^{* * *} \\
(4.00)\end{array}$ & $\begin{array}{c}0.028^{* * * *} \\
(4.09)\end{array}$ \\
\hline$B O R D$ & $\begin{array}{l}0.033 \\
(1.59)\end{array}$ & $\begin{array}{l}0.034 \\
(1.61)\end{array}$ & $\begin{array}{l}0.035^{*} \\
(1.65)\end{array}$ & $\begin{array}{l}0.033 \\
(1.59)\end{array}$ & $\begin{array}{l}0.033 \\
(1.59)\end{array}$ & $\begin{array}{l}0.033 \\
(1.57)\end{array}$ \\
\hline$D U A L$ & $\begin{array}{c}-0.019 \text { ** } \\
(-2.10)\end{array}$ & $\begin{array}{c}-0.019^{* *} \\
(-2.11)\end{array}$ & $\begin{array}{c}-0.019 \text { ** } \\
(-2.14)\end{array}$ & $\begin{array}{c}-0.019^{* * *} \\
(-2.07)\end{array}$ & $\begin{array}{c}-0.018 \text { ** } \\
(-2.03)\end{array}$ & $\begin{array}{c}-0.018 \text { ** } \\
(-2.04)\end{array}$ \\
\hline TOP1 & $\begin{array}{l}0.021 \\
(0.70)\end{array}$ & $\begin{array}{l}0.021 \\
(0.73)\end{array}$ & $\begin{array}{l}0.022 \\
(0.74)\end{array}$ & $\begin{array}{l}0.023 \\
(0.78)\end{array}$ & $\begin{array}{l}0.023 \\
(0.77)\end{array}$ & $\begin{array}{l}0.023 \\
(0.78)\end{array}$ \\
\hline BIG4 & $\begin{array}{l}0.027 \\
(1.34)\end{array}$ & $\begin{array}{l}0.026 \\
(1.32)\end{array}$ & $\begin{array}{l}0.025 \\
(1.27)\end{array}$ & $\begin{array}{l}0.028 \\
(1.38)\end{array}$ & $\begin{array}{l}0.027 \\
(1.35)\end{array}$ & $\begin{array}{l}0.028 \\
(1.39)\end{array}$ \\
\hline OWNER & $\begin{array}{c}0.014^{* * * *} \\
(4.14)\end{array}$ & $\begin{array}{c}0.013^{* * * *} \\
(4.01)\end{array}$ & $\begin{array}{c}0.013^{* * *} \\
(3.90)\end{array}$ & $\begin{array}{c}0.013^{* * * *} \\
(4.00)\end{array}$ & $\begin{array}{c}0.013^{* * *} \\
(3.85)\end{array}$ & $\begin{array}{c}0.013^{* * *} \\
(3.84)\end{array}$ \\
\hline$G D P$ & $\begin{array}{l}0.075 \\
(0.69)\end{array}$ & $\begin{array}{l}0.086 \\
(0.79)\end{array}$ & $\begin{array}{l}0.029 \\
(0.27)\end{array}$ & $\begin{array}{l}0.092 \\
(0.85)\end{array}$ & $\begin{array}{l}0.114 \\
(1.05)\end{array}$ & $\begin{array}{l}0.086 \\
(0.79)\end{array}$ \\
\hline$M K T$ & $\begin{array}{c}0.026^{* * * *} \\
(3.55)\end{array}$ & $\begin{array}{c}0.024^{* * * *} \\
(3.17)\end{array}$ & $\begin{array}{c}0.022^{* * *} \\
(3.02)\end{array}$ & $\begin{array}{c}0.027^{* * * *} \\
(3.61)\end{array}$ & $\begin{array}{c}0.026^{* * * *} \\
(3.43)\end{array}$ & $\begin{array}{c}0.028^{* * * *} \\
(3.68)\end{array}$ \\
\hline Intercept & $\begin{array}{c}-0.240 * * * \\
(-3.73)\end{array}$ & $\begin{array}{c}-0.245^{* * *} \\
(-3.78)\end{array}$ & $\begin{array}{c}-0.245^{* * *} \\
(-3.82)\end{array}$ & $\begin{array}{c}-0.249^{* * *} \\
(-3.84)\end{array}$ & $\begin{array}{c}-0.270^{\text {*** }} \\
(-4.13)\end{array}$ & $\begin{array}{c}-0.238^{* * *} \\
(-3.52)\end{array}$ \\
\hline Year E Ind. & Yes & Yes & Yes & Yes & Yes & Yes \\
\hline$N$ & 7227 & 7227 & 7227 & 7227 & 7227 & 7227 \\
\hline Adj. $R^{2}$ & 0.147 & 0.146 & 0.147 & 0.146 & 0.146 & 0.146 \\
\hline
\end{tabular}

$* * *$, and ${ }^{* * *}$ indicate statistical significance at the $10 \%, 5 \%$, and $1 \%$ levels, respectively. The $t$-values are shown in parentheses. The standard errors are clustered at the firm level.

\subsection{Further Analysis on the Cross-Sectional Variations}

\subsubsection{Analysis Conditional on Ownership Features}

We found that the effect of air pollution on the quality of a firm's internal control varies under different structures of ownership. In column (1) of Table 6, we examine whether the type of ultimate 
owner is a factor driving cross-sectional variation. For state-owned enterprises (SOEs), constructing and maintaining an effective internal control system are not only standards part of business, but also an important political mission echoing the government's call to improve the capability needed to manage systematic risk (e.g., [49]). Another consideration is that the positions of chairman and CEO in SOEs have a political rank. Therefore, these enterprises are less likely to respond to air pollution by reducing their level of internal control; they are more likely to tolerate its negative effects. The significantly negative coefficient of $A P \times S O E$ confirms our prediction by showing that the negative effect of air pollution on internal control quality is significantly lower in SOEs and non-SOEs. In column (2), we test whether the ownership concentration of shares moderates the effect found in the main test. As the shareholding of the controlling owner increases, he/she will be more sensitive to risk and thus have higher incentive to increase internal control [50]. The results show that the coefficient of $A P \times C O N T S H R$ is significantly positive at the $5 \%$ level, supporting the proposition that the negative effects of air pollution is mitigated when the corporate ownership is concentrated, i.e., when there is incentive for good internal control quality.

\subsubsection{Analysis of the External Environment}

We take two types of external environment, the industrial operating cycle and the market pressure resulting from product competition. The industrial operating cycle affects corporate decisions because firms in different stages face different kinds of risk and opportunity (e.g., [51-54]). Firms in the downward cycle are exposed to high level of external risk and uncertainty, requiring a sound internal control system and effective practices. This creates pressure on the owners and the management to implement and maintain internal control systems that are rational, prudent, and reduce the effects of negative emotions incurred by polluted air. We define downward cycle (INDOWN) as an indicator equal to 1 if the weighted market returns of all firms in that industry-year are negative, and zero otherwise. The results in column (1) of Table 7 support the above reasoning by showing that the coefficient of $A P \times I N D O W N$ is significantly positive, indicating that the negative effect of air pollution on internal control is small when the industry as a whole is in a downward cycle.

In column (2) of Table 7, we investigate the role of market pressure. The literature shows that highly competitive market can promote the improvement of internal control because effective controls in turn help the firm gain a competitive advantage (e.g., [55-57]). Thus, managers in the competitive markets have a strong incentive to overcome the negative emotions exacerbated by polluted air. Following research $[58,59]$, we use the price-cost margin $(P C M)$, or the Lerner Index, as a measure market pressure. Its higher value indicates less competition from industry peers. The significantly positive coefficient of $A P \times P C M$ provides evidence for our prediction.

\subsubsection{Analysis Conditional on the Chairman's Demographic Characteristics}

Given that the Standard and the Guidelines stipulate that the chairman of the board of directors is responsible for internal control, the chairman's traits should moderate our finding that the effect of air pollution on internal control quality varies under different ownership structures. We focus on two widely explored demographic characteristics, gender and tenure, as shown in Table 8. Females are generally calmer, more conservative, and more moral than males (e.g., [60,61]). Therefore, a female chairman may better suppress the negative emotions induced by air pollution and pay more attention to monitoring and supervising internal control in a bad environment, thereby reducing the negative impact of air pollution on internal control. This is confirmed by the significantly positive coefficient of $A P \times F E M A L$ in column (1). In terms of tenure, studies show that longer tenure helps form specific experience and knowledge (e.g., [62]), giving the chairman a deeper understanding of the business environment, and bringing rich personal experience to bear on resolving challenges. Thus, a chairman with longer tenure is less likely to be adversely affected by air pollution, which is supported by the significantly positive coefficient of $A P \times T E N U R E$ in column (2). 
Table 6. Cross-sectional Tests on Ownership Features.

\begin{tabular}{|c|c|c|}
\hline & (1) & (2) \\
\hline Dep. Variable $=$ & IC & IC \\
\hline$A P$ & $\begin{array}{c}-0.073^{* * *} \\
(-3.05)\end{array}$ & $\begin{array}{c}-0.143 * * * \\
(-3.15)\end{array}$ \\
\hline$A P \times S O E$ & $\begin{array}{c}0.086^{* *} \\
(2.37)\end{array}$ & \\
\hline$S O E$ & $\begin{array}{c}-0.082^{* *} \\
(-2.21)\end{array}$ & \\
\hline$A P \times C O N T S H R$ & & $\begin{array}{c}0.003 * * \\
(2.41)\end{array}$ \\
\hline CONTSHR & & $\begin{array}{l}-0.002 \\
(-1.58)\end{array}$ \\
\hline SIZE & $\begin{array}{c}0.071^{* * *} \\
(13.52)\end{array}$ & $\begin{array}{c}0.073^{* * *} \\
(13.44)\end{array}$ \\
\hline$R O A$ & $\begin{array}{l}-0.000 \\
(-0.01)\end{array}$ & $\begin{array}{l}-0.004 \\
(-0.06)\end{array}$ \\
\hline LEV & $\begin{array}{c}-0.189 * * * \\
(-7.31)\end{array}$ & $\begin{array}{c}-0.184^{* * *} \\
(-6.95)\end{array}$ \\
\hline SEO & $\begin{array}{c}-0.084 * * \\
(-2.21)\end{array}$ & $\begin{array}{c}-0.084^{* *} \\
(-2.18)\end{array}$ \\
\hline GROW & $\begin{array}{c}-0.039^{* * *} \\
(-6.49)\end{array}$ & $\begin{array}{c}-0.040 * * * \\
(-6.64)\end{array}$ \\
\hline INVE & $\begin{array}{c}0.080^{* *} \\
(2.13)\end{array}$ & $\begin{array}{l}0.073 * \\
(1.94)\end{array}$ \\
\hline$M A$ & $\begin{array}{l}-0.003 \\
(-0.44)\end{array}$ & $\begin{array}{l}-0.001 \\
(-0.14)\end{array}$ \\
\hline SEGM & $\begin{array}{c}0.017 \text { *** } \\
(3.22)\end{array}$ & $\begin{array}{c}0.017^{* * *} \\
(3.14)\end{array}$ \\
\hline EXPO & $\begin{array}{l}0.003 \\
(0.35)\end{array}$ & $\begin{array}{l}0.007 \\
(0.84)\end{array}$ \\
\hline$A G E$ & $\begin{array}{c}0.027 * * * \\
(3.91)\end{array}$ & $\begin{array}{c}0.028^{* * *} \\
(3.97)\end{array}$ \\
\hline$B O R D$ & $\begin{array}{l}0.036^{*} \\
(1.72)\end{array}$ & $\begin{array}{l}0.041 * \\
(1.90)\end{array}$ \\
\hline DUAL & $\begin{array}{c}-0.020 * * \\
(-2.19)\end{array}$ & $\begin{array}{c}-0.018 * \\
(-1.96)\end{array}$ \\
\hline TOP1 & $\begin{array}{l}0.019 \\
(0.66)\end{array}$ & $\begin{array}{l}-0.028 \\
(-0.46)\end{array}$ \\
\hline BIG4 & $\begin{array}{l}0.027 \\
(1.37)\end{array}$ & $\begin{array}{l}0.028 \\
(1.39)\end{array}$ \\
\hline OWNER & $\begin{array}{l}0.016^{* *} \\
(2.00)\end{array}$ & $\begin{array}{c}0.012 * * * \\
(3.59)\end{array}$ \\
\hline GDP & $\begin{array}{l}0.070 \\
(0.65)\end{array}$ & $\begin{array}{l}0.095 \\
(0.87)\end{array}$ \\
\hline$M K T$ & $\begin{array}{c}0.024^{* * *} \\
(3.26)\end{array}$ & $\begin{array}{c}0.024^{* * *} \\
(3.19)\end{array}$ \\
\hline Intercept & $\begin{array}{c}-0.213^{* * *} \\
(-3.18)\end{array}$ & $\begin{array}{c}-0.184^{* *} \\
(-2.47)\end{array}$ \\
\hline Year E Ind. & Yes & Yes \\
\hline$N$ & 7227 & 6938 \\
\hline Adj. $R^{2}$ & 0.147 & 0.151 \\
\hline
\end{tabular}

$* * *$, and $* * *$ indicate statistical significance at the $10 \%, 5 \%$, and $1 \%$ levels, respectively. The $t$-values are shown in parentheses. The standard errors are clustered at the firm level. 
Table 7. Cross-sectional Tests on External Environment.

\begin{tabular}{|c|c|c|}
\hline & (1) & (2) \\
\hline Dep. Variable $=$ & IC & IC \\
\hline$A P$ & $\begin{array}{c}-0.062 * * * \\
(-2.93)\end{array}$ & $\begin{array}{c}-0.050 * * \\
(-2.55)\end{array}$ \\
\hline$A P \times I N D O W N$ & $\begin{array}{c}0.092 * * \\
(2.55)\end{array}$ & \\
\hline INDOWN & $\begin{array}{l}-0.049 \\
(-1.56)\end{array}$ & \\
\hline$A P \times P C M$ & & $\begin{array}{c}0.093 * \\
(1.72)\end{array}$ \\
\hline$P C M$ & & $\begin{array}{l}-0.037 \\
(-0.67)\end{array}$ \\
\hline SIZE & $\begin{array}{c}0.072 * * * \\
(13.51)\end{array}$ & $\begin{array}{c}0.070 * * * \\
(13.15)\end{array}$ \\
\hline$R O A$ & $\begin{array}{l}0.004 \\
(0.04)\end{array}$ & $\begin{array}{l}-0.068 \\
(-0.79)\end{array}$ \\
\hline$L E V$ & $\begin{array}{c}-0.186^{* * *} \\
(-7.16)\end{array}$ & $\begin{array}{c}-0.187^{* * *} \\
(-7.19)\end{array}$ \\
\hline SEO & $\begin{array}{c}-0.081 \text { ** } \\
(-2.14)\end{array}$ & $\begin{array}{c}-0.088^{* *} \\
(-2.31)\end{array}$ \\
\hline GROW & $\begin{array}{c}-0.038 \text { *** } \\
(-6.42)\end{array}$ & $\begin{array}{c}-0.040^{* * *} \\
(-6.57)\end{array}$ \\
\hline$I N V E$ & $\begin{array}{c}0.078^{* *} \\
(2.07)\end{array}$ & $\begin{array}{c}0.069 * \\
(1.83)\end{array}$ \\
\hline$M A$ & $\begin{array}{l}-0.004 \\
(-0.48)\end{array}$ & $\begin{array}{l}-0.004 \\
(-0.56)\end{array}$ \\
\hline SEGM & $\begin{array}{c}0.018^{* * *} \\
(3.24)\end{array}$ & $\begin{array}{c}0.018^{* * *} \\
(3.39)\end{array}$ \\
\hline EXPO & $\begin{array}{l}0.002 \\
(0.23)\end{array}$ & $\begin{array}{l}0.003 \\
(0.34)\end{array}$ \\
\hline$A G E$ & $\begin{array}{c}0.027^{* * *} \\
(3.86)\end{array}$ & $\begin{array}{c}0.028^{* * *} \\
(4.04)\end{array}$ \\
\hline$B O R D$ & $\begin{array}{l}0.033 \\
(1.59)\end{array}$ & $\begin{array}{c}0.035^{*} \\
(1.65)\end{array}$ \\
\hline DUAL & $\begin{array}{c}-0.019 * * \\
(-2.08)\end{array}$ & $\begin{array}{c}-0.019 * * \\
(-2.15)\end{array}$ \\
\hline TOP1 & $\begin{array}{l}0.021 \\
(0.70) \\
\end{array}$ & $\begin{array}{l}0.021 \\
(0.71)\end{array}$ \\
\hline BIG4 & $\begin{array}{l}0.026 \\
(1.32)\end{array}$ & $\begin{array}{l}0.030 \\
(1.49)\end{array}$ \\
\hline OWNER & $\begin{array}{c}0.014^{* * *} \\
(4.13)\end{array}$ & $\begin{array}{c}0.014^{* * *} \\
(4.12)\end{array}$ \\
\hline GDP & $\begin{array}{l}0.080 \\
(0.73)\end{array}$ & $\begin{array}{l}0.083 \\
(0.76)\end{array}$ \\
\hline$M K T$ & $\begin{array}{c}0.025^{* * *} \\
(3.45)\end{array}$ & $\begin{array}{c}0.025^{* * *} \\
(3.42)\end{array}$ \\
\hline Intercept & $\begin{array}{c}-0.218^{* * *} \\
(-3.32)\end{array}$ & $\begin{array}{c}-0.223^{* * *} \\
(-3.41)\end{array}$ \\
\hline Year E Ind. & Yes & Yes \\
\hline$N$ & 7227 & 7182 \\
\hline Adj. $R^{2}$ & 0.147 & 0.148 \\
\hline
\end{tabular}

$*, * *$, and $* * *$ indicate statistical significance at the $10 \%, 5 \%$, and $1 \%$ levels, respectively. The $t$-values are shown in parentheses. The standard errors are clustered at the firm level. 
Table 8. Cross-sectional Tests on the Chairman's Demographic Characteristics.

\begin{tabular}{|c|c|c|}
\hline & (1) & (2) \\
\hline Dep. Variable $=$ & IC & IC \\
\hline$A P$ & $\begin{array}{c}-0.046^{* *} \\
(-2.39)\end{array}$ & $\begin{array}{c}-0.084^{* * *} \\
(-2.61)\end{array}$ \\
\hline$A P \times F E M A L E$ & $\begin{array}{c}0.134 * \\
(1.70)\end{array}$ & \\
\hline FEMALE & $\begin{array}{c}-0.161 * * \\
(-2.46)\end{array}$ & \\
\hline$A P \times T E N U R E$ & & $\begin{array}{c}0.006^{*} \\
(1.71)\end{array}$ \\
\hline TENURE & & $\begin{array}{l}-0.001 \\
(-0.26)\end{array}$ \\
\hline SIZE & $\begin{array}{c}0.071 \text { *** } \\
(13.51)\end{array}$ & $\begin{array}{c}0.069^{* * *} \\
(13.03)\end{array}$ \\
\hline$R O A$ & $\begin{array}{l}0.000 \\
(0.01)\end{array}$ & $\begin{array}{l}-0.023 \\
(-0.29)\end{array}$ \\
\hline$L E V$ & $\begin{array}{c}-0.189^{* * *} \\
(-7.29)\end{array}$ & $\begin{array}{c}-0.184^{* * *} \\
(-7.15)\end{array}$ \\
\hline SEO & $\begin{array}{c}-0.082 \text { ** } \\
(-2.16)\end{array}$ & $\begin{array}{c}-0.081 \text { ** } \\
(-2.14)\end{array}$ \\
\hline GROW & $\begin{array}{c}-0.039^{* * *} \\
(-6.54)\end{array}$ & $\begin{array}{c}-0.035^{* * * *} \\
(-5.87)\end{array}$ \\
\hline INVE & $\begin{array}{c}0.080 * * \\
(2.14)\end{array}$ & $\begin{array}{c}0.078^{* *} \\
(2.11)\end{array}$ \\
\hline$M A$ & $\begin{array}{l}-0.003 \\
(-0.42)\end{array}$ & $\begin{array}{l}-0.003 \\
(-0.40)\end{array}$ \\
\hline SEGM & $\begin{array}{c}0.018^{* * *} \\
(3.26)\end{array}$ & $\begin{array}{c}0.016^{* * *} \\
(2.92)\end{array}$ \\
\hline EXPO & $\begin{array}{l}0.002 \\
(0.25) \\
\end{array}$ & $\begin{array}{l}0.002 \\
(0.18)\end{array}$ \\
\hline$A G E$ & $\begin{array}{c}0.027 * * * \\
(3.97)\end{array}$ & $\begin{array}{c}0.015^{* *} \\
(2.01)\end{array}$ \\
\hline$B O R D$ & $\begin{array}{l}0.033 \\
(1.59)\end{array}$ & $\begin{array}{l}0.031 \\
(1.48)\end{array}$ \\
\hline$D U A L$ & $\begin{array}{c}-0.019 \text { ** } \\
(-2.14)\end{array}$ & $\begin{array}{c}-0.020 * * \\
(-2.28)\end{array}$ \\
\hline TOP1 & $\begin{array}{l}0.023 \\
(0.78)\end{array}$ & $\begin{array}{l}0.030 \\
(1.01)\end{array}$ \\
\hline BIG4 & $\begin{array}{l}0.027 \\
(1.32)\end{array}$ & $\begin{array}{l}0.030 \\
(1.50)\end{array}$ \\
\hline OWNER & $\begin{array}{c}0.013^{* * *} \\
(3.95)\end{array}$ & $\begin{array}{c}0.015^{* * *} \\
(4.39)\end{array}$ \\
\hline GDP & $\begin{array}{l}0.080 \\
(0.73)\end{array}$ & $\begin{array}{l}0.064 \\
(0.59)\end{array}$ \\
\hline$M K T$ & $\begin{array}{c}0.026^{* * *} \\
(3.50)\end{array}$ & $\begin{array}{c}0.025^{* * *} \\
(3.41)\end{array}$ \\
\hline Intercept & $\begin{array}{c}-0.229^{* * *} \\
(-3.51)\end{array}$ & $\begin{array}{c}-0.182 * * * \\
(-2.68)\end{array}$ \\
\hline Year E Ind. & Yes & Yes \\
\hline$N$ & 7225 & 7225 \\
\hline Adj. $R^{2}$ & 0.148 & 0.150 \\
\hline
\end{tabular}

$*, * *$, and $* * *$ indicate statistical significance at the $10 \%, 5 \%$, and $1 \%$ levels, respectively. The $t$-values are shown in parentheses. The standard errors are clustered at the firm level. 


\subsubsection{Analysis Conditional on Employee-Related Factors}

Our main argument relies on the assumption that air pollution, via individual psychological status, affects the quality of internal control, which is a standard process involving the participation not only of a chairman but also of all employees. Therefore, work intensity and employee quality can become direct moderating variables. Firms with more employees may be more strongly influenced by air pollution. Consequently our revealed negative effect intensifies in these firms, as indicated by the significantly negative coefficient of $A P \times L A B I N T$ in column (1) of Table 9, where LABINT is an indicator that equals 1 if the work intensity (i.e., the number of employees/sales to measures) is higher than industry median, and 0 otherwise. Highly educated employees usually function at a higher psychological level when coping with environmental change, in that they can still maintain their normal routine and facilitate the implementation of internal control measures. Therefore, as indicated by the significantly positive coefficient of $A P \times E M P E D U$ in column (2) of Table 9, where EMPEDU equals 1 if the firm's proportion of employees holding at least a Bachelor's degree is higher than its industry mean, and 0 otherwise, then the negative effect of air pollution on internal control quality weakens.

\subsection{The Heavy Pollution Industry}

A potential endogenous concern in this paper is that firms located in polluted cities inherently have lower internal control quality than those in non-polluted cities. To exclude this concern, we perform a cross-section test based on the severity of industry pollution. Specifically, based on the "Catalogue of Reclassification Administration of Environmental Protection Inspection Enterprises among Listed Companies" issued by Chinese Ministry of Environmental Protection in 2008, we partition all industries into two groups-pollution and non-pollution. Accordingly, companies in the 16 polluted industries are classified as the "Pollution" sub-sample and the rest as the "Non-Pollution". From Panel A of Table 10, we can see from the descriptive statistics that companies in the "Pollution" sub-sample have higher IC. In Panel B of Table 10, we interact $A P$ with Pollution. The positive coefficient on the interaction term indicates that in industries with pollution, the negative association between IC and AP is less pronounced than in industries with no pollution. Therefore, our results are not affected by the omitted geographic variables.

\subsection{The Institutional Cost of Air Pollution: Test on Other Economic Consequences}

In this section, we test the additional economic consequences of air pollution possibly induced by the deterioration of internal control. Table 11 shows that three internal control quality influenced accounting information quality, agency costs, and firm value (e.g., $[22,63,64])$, which are respectively proxied by Khan and Watts's [65] accounting conservatism score (C-SCORE), perquisite consumption (PERK), measured as the sum of perks scaled by operating income, and firm value, measured by the accumulated buy-and-hold abnormal returns over the 12 months of a fiscal year (BHAR). Consistent with our predictions, the results show that air pollution can lead to less conservative accounting information, more benefits enjoyed by insiders, and consequently lower firm value. 
Table 9. Cross-sectional Tests on Employee-related Factors.

\begin{tabular}{|c|c|c|}
\hline & (1) & (2) \\
\hline Dep. Variable $=$ & IC & IC \\
\hline$A P$ & $\begin{array}{c}-0.064^{* *} \\
(-2.20)\end{array}$ & $\begin{array}{c}-0.079 * * * \\
(-3.14)\end{array}$ \\
\hline$A P \times L A B I N T$ & $\begin{array}{c}-0.045^{*} \\
(-1.80)\end{array}$ & \\
\hline LABINT & $\begin{array}{l}-0.017 \\
(-0.86)\end{array}$ & \\
\hline$A P \times E M P E D U$ & & $\begin{array}{l}0.058 * \\
(1.77)\end{array}$ \\
\hline EMPEDU & & $\begin{array}{l}-0.018 \\
(-0.63)\end{array}$ \\
\hline SIZE & $\begin{array}{c}0.068^{* * *} \\
(16.46)\end{array}$ & $\begin{array}{c}0.071^{* * *} \\
(13.41)\end{array}$ \\
\hline$R O A$ & $\begin{array}{l}-0.001 \\
(-0.01)\end{array}$ & $\begin{array}{l}-0.016 \\
(-0.20)\end{array}$ \\
\hline LEV & $\begin{array}{c}-0.189 * * * \\
(-9.51)\end{array}$ & $\begin{array}{c}-0.184^{* * *} \\
(-7.13)\end{array}$ \\
\hline SEO & $\begin{array}{c}-0.079 * * \\
(-2.15)\end{array}$ & $\begin{array}{c}-0.079 * * \\
(-2.08)\end{array}$ \\
\hline GROW & $\begin{array}{c}-0.038^{* * *} \\
(-7.01)\end{array}$ & $\begin{array}{c}-0.039^{* * * *} \\
(-6.61)\end{array}$ \\
\hline$I N V E$ & $\begin{array}{c}0.082 * * * \\
(2.87)\end{array}$ & $\begin{array}{c}0.078^{* *} \\
(2.08)\end{array}$ \\
\hline$M A$ & $\begin{array}{l}-0.003 \\
(-0.42)\end{array}$ & $\begin{array}{l}-0.003 \\
(-0.40)\end{array}$ \\
\hline SEGM & $\begin{array}{c}0.016^{* * *} \\
(3.68)\end{array}$ & $\begin{array}{c}0.017^{* * *} \\
(3.16)\end{array}$ \\
\hline EXPO & $\begin{array}{l}0.002 \\
(0.28)\end{array}$ & $\begin{array}{l}0.005 \\
(0.55)\end{array}$ \\
\hline$A G E$ & $\begin{array}{c}0.026^{* * * *} \\
(3.06)\end{array}$ & $\begin{array}{c}0.028^{* * *} \\
(4.01)\end{array}$ \\
\hline$B O R D$ & $\begin{array}{l}0.031 * \\
(1.71)\end{array}$ & $\begin{array}{l}0.033 \\
(1.58)\end{array}$ \\
\hline$D U A L$ & $\begin{array}{c}-0.019 \text { ** } \\
(-2.51)\end{array}$ & $\begin{array}{c}-0.020 \text { ** } \\
(-2.26)\end{array}$ \\
\hline TOP1 & $\begin{array}{l}0.017 \\
(0.78)\end{array}$ & $\begin{array}{l}0.024 \\
(0.82)\end{array}$ \\
\hline BIG4 & $\begin{array}{l}0.028 * \\
(1.80)\end{array}$ & $\begin{array}{l}0.024 \\
(1.21)\end{array}$ \\
\hline OWNER & $\begin{array}{c}0.014^{* * *} \\
(4.37)\end{array}$ & $\begin{array}{c}0.013^{* * *} \\
(3.80)\end{array}$ \\
\hline GDP & $\begin{array}{l}0.087 \\
(0.93)\end{array}$ & $\begin{array}{l}0.082 \\
(0.76)\end{array}$ \\
\hline$M K T$ & $\begin{array}{c}0.026^{* * *} \\
(3.51)\end{array}$ & $\begin{array}{c}0.025 * * * \\
(3.39)\end{array}$ \\
\hline Intercept & $\begin{array}{c}-0.188^{* * *} \\
(-2.80)\end{array}$ & $\begin{array}{c}-0.212^{* * *} \\
(-3.21)\end{array}$ \\
\hline Year E Ind. & Yes & Yes \\
\hline$N$ & 7225 & 7225 \\
\hline Adj. $R^{2}$ & 0.148 & 0.150 \\
\hline
\end{tabular}

$*, * *$, and $* * *$ indicate statistical significance at the $10 \%, 5 \%$, and $1 \%$ levels, respectively. The $t$-values are shown in parentheses. The standard errors are clustered at the firm level. 
Table 10. The Heavy Pollution Industry Test.

\begin{tabular}{|c|c|c|c|}
\hline \multicolumn{4}{|c|}{ Panel A: Descriptive Statistics for The Heavy Pollution Industry } \\
\hline Variables & $\begin{array}{l}\text { Non-Pollution } \\
(\mathrm{Obs}=4582)\end{array}$ & Pollution $(\mathrm{Obs}=2635)$ & Dif. \\
\hline IC & 0.503 & 0.522 & $-0.018^{* * *}$ \\
\hline$A P$ & 0.813 & 0.842 & $-0.029^{* * *}$ \\
\hline SIZE & 8.350 & 8.431 & $-0.082^{* * *}$ \\
\hline$R O A$ & 0.047 & 0.053 & $-0.005^{* * *}$ \\
\hline LEV & 0.439 & 0.421 & $0.018^{* * *}$ \\
\hline SEO & 0.042 & 0.036 & $0.006^{* * *}$ \\
\hline GROW & 0.237 & 0.111 & $0.126^{* * *}$ \\
\hline$I N V E$ & 0.163 & 0.119 & $0.044^{* * *}$ \\
\hline$M A$ & 0.327 & 0.271 & $0.057^{* * *}$ \\
\hline SEGM & 2.459 & 2.326 & $0.134^{* * *}$ \\
\hline EXPO & 0.270 & 0.308 & $-0.038^{* * *}$ \\
\hline$A G E$ & 2.183 & 2.272 & $-0.089^{* * *}$ \\
\hline$B O R D$ & 2.118 & 2.146 & $-0.029^{* * *}$ \\
\hline$D U A L$ & 0.277 & 0.235 & $0.042 * * *$ \\
\hline TOP1 & 0.340 & 0.352 & $-0.012^{* * *}$ \\
\hline BIG4 & 0.054 & 0.055 & -0.001 \\
\hline LEVE & 2.066 & 2.165 & $-0.098^{* * *}$ \\
\hline GDP & 0.081 & 0.073 & $0.008^{* * *}$ \\
\hline MKT & 1.740 & 1.502 & $0.237 * * *$ \\
\hline \multicolumn{4}{|c|}{ Panel B: Cross-sectional Test for Heavy Pollution Industry } \\
\hline \multicolumn{2}{|c|}{ Dep.Variable $=$} & \multicolumn{2}{|c|}{ IC } \\
\hline \multicolumn{2}{|c|}{$A P$} & \multicolumn{2}{|c|}{$\begin{array}{c}-0.055 * \\
(-1.75)\end{array}$} \\
\hline \multicolumn{2}{|c|}{ AP $\times$ Pollution } & \multicolumn{2}{|c|}{$0.039 *$} \\
\hline \multicolumn{2}{|c|}{ Pollution } & & \\
\hline \multicolumn{2}{|c|}{ SIZE } & \multicolumn{2}{|c|}{$\begin{array}{l}-0.003 \\
(-0.05)\end{array}$} \\
\hline \multicolumn{2}{|c|}{$R O A$} & \multicolumn{2}{|c|}{$-0.187^{* * *}$} \\
\hline \multicolumn{2}{|c|}{$L E V$} & \multicolumn{2}{|c|}{$\begin{array}{c}-0.081 \text { ** } \\
(-2.21)\end{array}$} \\
\hline \multicolumn{2}{|c|}{ SEO } & \multicolumn{2}{|c|}{$(-7.00)$} \\
\hline \multicolumn{2}{|c|}{ GROW } & & \\
\hline & & & \\
\hline & & & \\
\hline & & & \\
\hline & & & \\
\hline & & & \\
\hline & & & \\
\hline & & & \\
\hline & & & \\
\hline & & & \\
\hline & & & \\
\hline & & & \\
\hline & & & \\
\hline & & & \\
\hline & & & \\
\hline & & & \\
\hline & & & \\
\hline
\end{tabular}

$* * *$, and ${ }^{* * *}$ indicate statistical significance at the $10 \%, 5 \%$, and $1 \%$ levels, respectively. The $z$-values are shown in the parentheses. The standard errors are clustered at the firm level. 
Table 11. Test on the Other Economic Consequences of Air Pollution.

\begin{tabular}{|c|c|c|c|}
\hline & (1) & (2) & (3) \\
\hline & Information Quality & $\begin{array}{l}\text { Perquisite } \\
\text { Consumption }\end{array}$ & Firm Value \\
\hline Dep. Variable $=$ & C-SCORE & PERK & $B H A R$ \\
\hline$A P$ & $\begin{array}{c}-0.002 * * \\
(-2.46)\end{array}$ & $\begin{array}{l}0.004^{*} \\
(1.69)\end{array}$ & $\begin{array}{c}-0.051 \text { ** } \\
(-2.14)\end{array}$ \\
\hline SIZE & $\begin{array}{c}0.017^{* * *} \\
(43.32)\end{array}$ & $\begin{array}{c}-0.004 * * * \\
(-5.76)\end{array}$ & $\begin{array}{c}-0.088 \text { *** } \\
(-12.04)\end{array}$ \\
\hline$R O A$ & $\begin{array}{l}-0.007 \\
(-1.20)\end{array}$ & $\begin{array}{c}-0.046^{* * *} \\
(-5.65)\end{array}$ & $\begin{array}{c}0.465^{* * *} \\
(3.70)\end{array}$ \\
\hline LEV & $\begin{array}{l}0.004 \\
(1.51)\end{array}$ & $\begin{array}{c}-0.008^{* * *} \\
(-2.75)\end{array}$ & $\begin{array}{c}0.268^{* * *} \\
(7.36)\end{array}$ \\
\hline SEO & $\begin{array}{l}0.003^{*} \\
(1.65)\end{array}$ & $\begin{array}{c}-0.005^{* *} \\
(-2.57)\end{array}$ & $\begin{array}{c}0.363^{* * *} \\
(5.09)\end{array}$ \\
\hline GROW & $\begin{array}{c}-0.001 \text { ** } \\
(-2.49)\end{array}$ & $\begin{array}{c}-0.002 * * * \\
(-3.71)\end{array}$ & $\begin{array}{c}0.093^{* * *} \\
(6.83)\end{array}$ \\
\hline INVE & $\begin{array}{l}-0.002 \\
(-0.68)\end{array}$ & $\begin{array}{l}-0.008 \\
(-0.97)\end{array}$ & $\begin{array}{l}-0.040 \\
(-0.80)\end{array}$ \\
\hline$M A$ & $\begin{array}{c}-0.001 * * \\
(-2.02)\end{array}$ & $\begin{array}{l}0.000 \\
(0.29)\end{array}$ & $\begin{array}{c}0.108^{* * *} \\
(7.44)\end{array}$ \\
\hline SEGM & $\begin{array}{c}-0.001 \text { ** } \\
(-2.41)\end{array}$ & $\begin{array}{l}0.001^{* *} \\
(2.45)\end{array}$ & $\begin{array}{c}-0.015^{* *} \\
(-2.02)\end{array}$ \\
\hline EXPO & $\begin{array}{c}-0.004^{* * *} \\
(-6.27)\end{array}$ & $\begin{array}{c}-0.002 * * * \\
(-3.57)\end{array}$ & $\begin{array}{c}-0.082^{* * *} \\
(-6.65)\end{array}$ \\
\hline$A G E$ & $\begin{array}{c}-0.001 * * * \\
(-3.78)\end{array}$ & $\begin{array}{c}0.002 \text { **** } \\
(2.58)\end{array}$ & $\begin{array}{c}-0.028^{* * *} \\
(-2.78)\end{array}$ \\
\hline BORD & $\begin{array}{l}0.002 \\
(1.29)\end{array}$ & $\begin{array}{l}0.000 \\
(0.07)\end{array}$ & $\begin{array}{l}-0.034 \\
(-1.25)\end{array}$ \\
\hline$D U A L$ & $\begin{array}{l}-0.000 \\
(-0.17)\end{array}$ & $\begin{array}{l}0.001 \\
(0.60)\end{array}$ & $\begin{array}{l}0.020 \\
(1.56)\end{array}$ \\
\hline TOP1 & $\begin{array}{l}0.001 \\
(0.74)\end{array}$ & $\begin{array}{c}-0.007 * * * \\
(-3.18)\end{array}$ & $\begin{array}{c}0.078^{* *} \\
(2.10)\end{array}$ \\
\hline BIG4 & $\begin{array}{l}-0.002 * \\
(-1.77)\end{array}$ & $\begin{array}{c}0.005^{* *} \\
(2.44)\end{array}$ & $\begin{array}{c}0.062^{* * *} \\
(2.74)\end{array}$ \\
\hline OWNER & $\begin{array}{c}0.000 * * \\
(2.17)\end{array}$ & $\begin{array}{c}-0.001 * * * \\
(-2.64)\end{array}$ & $\begin{array}{c}-0.008 \text { ** } \\
(-2.02)\end{array}$ \\
\hline$G D P$ & $\begin{array}{l}-0.004 \\
(-0.64) \\
\end{array}$ & $\begin{array}{l}0.006 \\
(0.35) \\
\end{array}$ & $\begin{array}{l}-0.012 \\
(-0.08) \\
\end{array}$ \\
\hline MKT & $\begin{array}{l}-0.001 \\
(-1.60)\end{array}$ & $\begin{array}{c}-0.002^{* * *} \\
(-3.10)\end{array}$ & $\begin{array}{l}0.011 \\
(1.33)\end{array}$ \\
\hline Intercept & $\begin{array}{c}-0.106^{* * *} \\
(-30.31)\end{array}$ & $\begin{array}{c}0.044^{* * *} \\
(8.01)\end{array}$ & $\begin{array}{c}0.689^{* * *} \\
(8.72)\end{array}$ \\
\hline Year $\mathcal{E}$ Ind. & Yes & Yes & Yes \\
\hline$N$ & 6718 & 7227 & 7209 \\
\hline Adj. $R^{2}$ & 0.567 & 0.084 & 0.301 \\
\hline
\end{tabular}

$*, * *$, and ${ }^{* * *}$ indicate statistical significance at the $10 \%, 5 \%$, and $1 \%$ levels, respectively. The $t$-values are shown in parentheses. The standard errors are clustered at the firm level.

\subsection{Regression Discontinuity Design}

To further mitigate the endogenous problem, we follow Chen et al. [66] and Ebenstein et al.'s paper [67], using the regression discontinuity design to reexamine the above result. During the 1950-1980 period of central planning, the Chinese government established free winter heating of homes and offices via the provision of free coal for fuel boilers as a basic right. The combustion of coal in boilers is associated with the release of air pollutants, and this right was only extended to areas located in North China, which is defined by the line formed by the Huai River and Qinling Mountain range. Thus, we follow Ebenstein et al.'s work [67], examine that whether audit quality exits discontinuous change for the heat policy line. We define the variable North as the latitude distance between a firm's 
headquarter and Huai River. A positive value means a firm's headquarter is located to the norther of Huai River and affected by the heating policy, and vice versa. We can see from Fig.1 that there is indeed a discontinuity around the 0 point, indicating that internal quality is affected by the air pollution caused by heating policy. In Table 12, we offer the RD result with a bandwidth of $2.45^{\circ}$ latitude. The non-parametric result is consistent with Figure 1.

Table 12. RD estimates of the impact of the Huai River Policy.

\begin{tabular}{|c|c|}
\hline Dep. Var. & $=I C$ \\
\hline \multicolumn{2}{|c|}{ Panel A: non-parametric test } \\
\hline North & $\begin{array}{c}-0.108^{* *} \\
(-2.25)\end{array}$ \\
\hline Control Variable & Yes \\
\hline \multicolumn{2}{|c|}{ Panel B: Local parameter OLS(Ordinary Least Squares) regression test } \\
\hline TREAT & $\begin{array}{c}-0.036^{*} \\
(-1.96)\end{array}$ \\
\hline SIZE & $\begin{array}{c}0.075 * * * \\
(10.09)\end{array}$ \\
\hline$R O A$ & $\begin{array}{l}-0.009 \\
(-0.08)\end{array}$ \\
\hline$L E V$ & $\begin{array}{c}-0.204^{* * *} \\
(-5.69)\end{array}$ \\
\hline SEO & $\begin{array}{l}-0.063 \\
(-0.95)\end{array}$ \\
\hline GROW & $\begin{array}{c}-0.030^{* * *} \\
(-3.15)\end{array}$ \\
\hline INVE & $\begin{array}{l}0.025 \\
(0.42)\end{array}$ \\
\hline$M A$ & $\begin{array}{l}0.010 \\
(0.77)\end{array}$ \\
\hline SEGM & $\begin{array}{l}0.010 \\
(1.32)\end{array}$ \\
\hline EXPO & $\begin{array}{l}-0.000 \\
(-0.03)\end{array}$ \\
\hline$A G E$ & $\begin{array}{c}0.017^{*} \\
(1.86)\end{array}$ \\
\hline BORD & $\begin{array}{l}0.060^{*} \\
(1.94)\end{array}$ \\
\hline DUAL & $\begin{array}{l}-0.011 \\
(-0.90)\end{array}$ \\
\hline TOP1 & $\begin{array}{l}-0.068 \\
(-1.62)\end{array}$ \\
\hline BIG4 & $\begin{array}{c}0.052^{* *} \\
(2.10)\end{array}$ \\
\hline LEVE & $\begin{array}{c}0.025 * * * \\
(5.53)\end{array}$ \\
\hline GDP & $\begin{array}{l}0.216 \\
(0.85)\end{array}$ \\
\hline MKT & $\begin{array}{c}0.031 \text { * } \\
(1.90)\end{array}$ \\
\hline Intercept & $\begin{array}{c}-0.297^{* * *} \\
(-2.95)\end{array}$ \\
\hline Year \& Ind. & Yes \\
\hline Bandwidth & $2.45^{\circ}$ \\
\hline N & 2299 \\
\hline $\operatorname{Adj} . R^{2}$ & 0.202 \\
\hline
\end{tabular}

$*, * *$, and ${ }^{* * *}$ indicate statistical significance at the $10 \%, 5 \%$, and $1 \%$ levels, respectively. The $z$-values are shown in the parentheses. The standard errors are clustered at the firm level.

\subsection{City Fixed Effect}

Another way to mitigate the omitted variable problem is to include the city fixed effects (or city-year fixed effects) in the main regression. As we can see from Table 13, results remain consistent after the inclusion of fixed effects. 


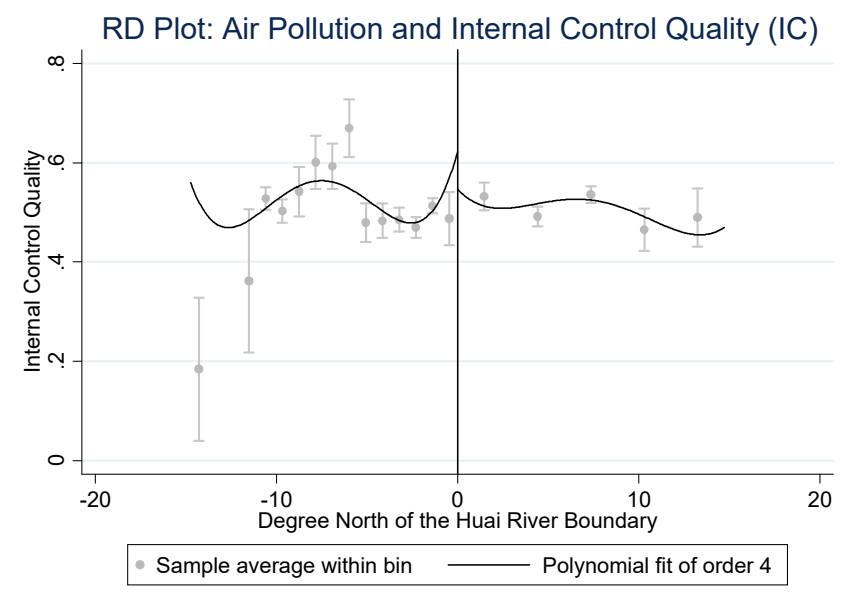

Figure 1. Internal Control Quality and Distance from the Huai River.

Table 13. Controlling City Fixed Effect.

\begin{tabular}{|c|c|c|}
\hline & (1) & (2) \\
\hline Dep. Variable= & IC & IC \\
\hline$A P$ & $\begin{array}{c}-0.062 * \\
(-1.69)\end{array}$ & $\begin{array}{c}-0.093 * \\
(-1.71)\end{array}$ \\
\hline SIZE & $\begin{array}{c}0.080^{* * *} \\
(16.31)\end{array}$ & $\begin{array}{c}0.075^{* * *} \\
(16.29)\end{array}$ \\
\hline$R O A$ & $\begin{array}{l}-0.112 \\
(-1.34)\end{array}$ & $\begin{array}{l}-0.086 \\
(-1.00)\end{array}$ \\
\hline$L E V$ & $\begin{array}{c}-0.191 * * * \\
(-8.77)\end{array}$ & $\begin{array}{c}-0.195^{* * *} \\
(-8.77)\end{array}$ \\
\hline SEO & $\begin{array}{c}-0.122 * * * \\
(-2.67)\end{array}$ & $\begin{array}{c}-0.118^{* *} \\
(-2.51)\end{array}$ \\
\hline GROW & $\begin{array}{c}-0.037^{* * *} \\
(-3.38)\end{array}$ & $\begin{array}{c}-0.034^{* * *} \\
(-3.03)\end{array}$ \\
\hline INVE & $\begin{array}{c}0.088^{* *} \\
(2.51)\end{array}$ & $\begin{array}{c}0.096^{* * *} \\
(2.67)\end{array}$ \\
\hline$M A$ & $\begin{array}{l}-0.003 \\
(-0.49)\end{array}$ & $\begin{array}{l}-0.005 \\
(-0.69)\end{array}$ \\
\hline SEGM & $\begin{array}{c}0.022 * * * \\
(4.54)\end{array}$ & $\begin{array}{c}0.021^{* * *} \\
(4.17)\end{array}$ \\
\hline EXPO & $\begin{array}{l}-0.001 \\
(-0.15)\end{array}$ & $\begin{array}{l}-0.002 \\
(-0.29)\end{array}$ \\
\hline$A G E$ & $\begin{array}{c}0.019^{* * * *} \\
(3.38)\end{array}$ & $\begin{array}{c}0.021 * * * \\
(3.59)\end{array}$ \\
\hline$B O R D$ & $\begin{array}{c}0.044^{* *} \\
(2.19)\end{array}$ & $\begin{array}{c}0.043^{* *} \\
(2.08)\end{array}$ \\
\hline DUAL & $\begin{array}{c}-0.016 \text { ** } \\
(-2.17)\end{array}$ & $\begin{array}{c}-0.017^{* *} \\
(-2.23)\end{array}$ \\
\hline TOP1 & $\begin{array}{l}0.013 \\
(0.53)\end{array}$ & $\begin{array}{l}0.009 \\
(0.34)\end{array}$ \\
\hline BIG4 & $\begin{array}{c}0.053^{* * *} \\
(3.66)\end{array}$ & $\begin{array}{c}0.038^{* *} \\
(2.53)\end{array}$ \\
\hline LEVE & $\begin{array}{c}0.016^{* * *} \\
(5.81)\end{array}$ & $\begin{array}{c}0.015^{* * *} \\
(5.61)\end{array}$ \\
\hline GDP & $\begin{array}{l}0.045 \\
(0.23)\end{array}$ & $\begin{array}{l}0.479 \\
(1.23)\end{array}$ \\
\hline$M K T$ & $\begin{array}{c}0.046^{* * *} \\
(2.85)\end{array}$ & $\begin{array}{c}0.041 \text { ** } \\
(2.40)\end{array}$ \\
\hline Intercept & $\begin{array}{c}-0.435^{* * *} \\
(-6.25)\end{array}$ & $\begin{array}{c}-0.447^{* * *} \\
(-5.70)\end{array}$ \\
\hline Ind & Yes & Yes \\
\hline Year & Yes & No \\
\hline City & Yes & No \\
\hline City $\times$ Year & No & Yes \\
\hline N & 7227 & 7227 \\
\hline Adj. $R^{2}$ & 0.166 & 0.150 \\
\hline
\end{tabular}

$* * *$, and ${ }^{* * *}$ indicate statistical significance at the $10 \%, 5 \%$, and $1 \%$ levels, respectively. The $z$-values are shown in the parentheses. The standard errors are clustered at the firm level. 


\subsection{Propensity Score Matched Sample}

In addition, we perform a cross-section test based on the severity of city pollution to further address the concern of omitted variables. Specifically, cites with above-median air pollution are classified as "High_Pollution" and below-median as "Low_Pollution". The concern caused by omitted variables problem is that firms in polluted regions inherently have worse internal control. However, in Panel A of Table 14, we can see from the comparison of descriptive statistics on the sub-samples of "High_Pollution" and "Low_Pollution" that firms in cities with high pollution have no different internal control quality from those in cities with low pollution. Nonetheless, we still employ the propensity score matching method to balance any potential inherent differences. The descriptive statistics for the PSM (Propensity Score Matched) sample show that there is no difference in firm characteristics in all respects after the matching procedure. More importantly, in Panel B of Table 14, we can see that our results are robust using the propensity score matching method. Therefore, we are confident that omitted geographic variable will not affect our findings.

\subsection{Robustness Tests}

\subsubsection{Alternative Sample and Measures}

Table 15 presents robustness checks using an alternative sample and alternative measures. First, to eliminate the possibility that our findings are overly affected by areas with extremely serious air pollution, we eliminate from the sample firms ranked in the top three provinces and top 15 cities in terms of air pollution, as shown in columns (1) and (2). Second, alternative measures of $I C$, the raw score of the Internal Control Index and the annual ranking of the score divided by 1000, are used in columns (3) and (4), respectively. Third, alternative measures of $A P$, the ranking of air quality for firms' home cities, and the proportion of polluted days in one year in those cities, are used in columns (5) and (6), respectively. Our main findings, as shown in Table 3, remain in all columns.

\subsubsection{Controlling for Climate}

Several studies have shown that climate (including temperature, wind, and weather) is an important factor affecting individual psychology and behavior (e.g., [68,69]), and we control for these factors in Table 16. HOT represents temperature, which is measured as the proportion of the days in a year when highest temperature exceeds $37^{\circ} \mathrm{C}$ in the firm's home city. WIND means the wind, which is measured as the number of days in a year when wind exceeds grade 3 in the home city. BAD is the weather status, which is measured as the proportion of days in a year when the weather is bad, with conditions being cloudy, rainy, or snowy, in the home city. Then, we conduct principal component analysis and integrate these three measures into one, CLIMATE. The results show that the coefficient of AP remains significantly negative in all columns. 
Table 14. PSM Sample.

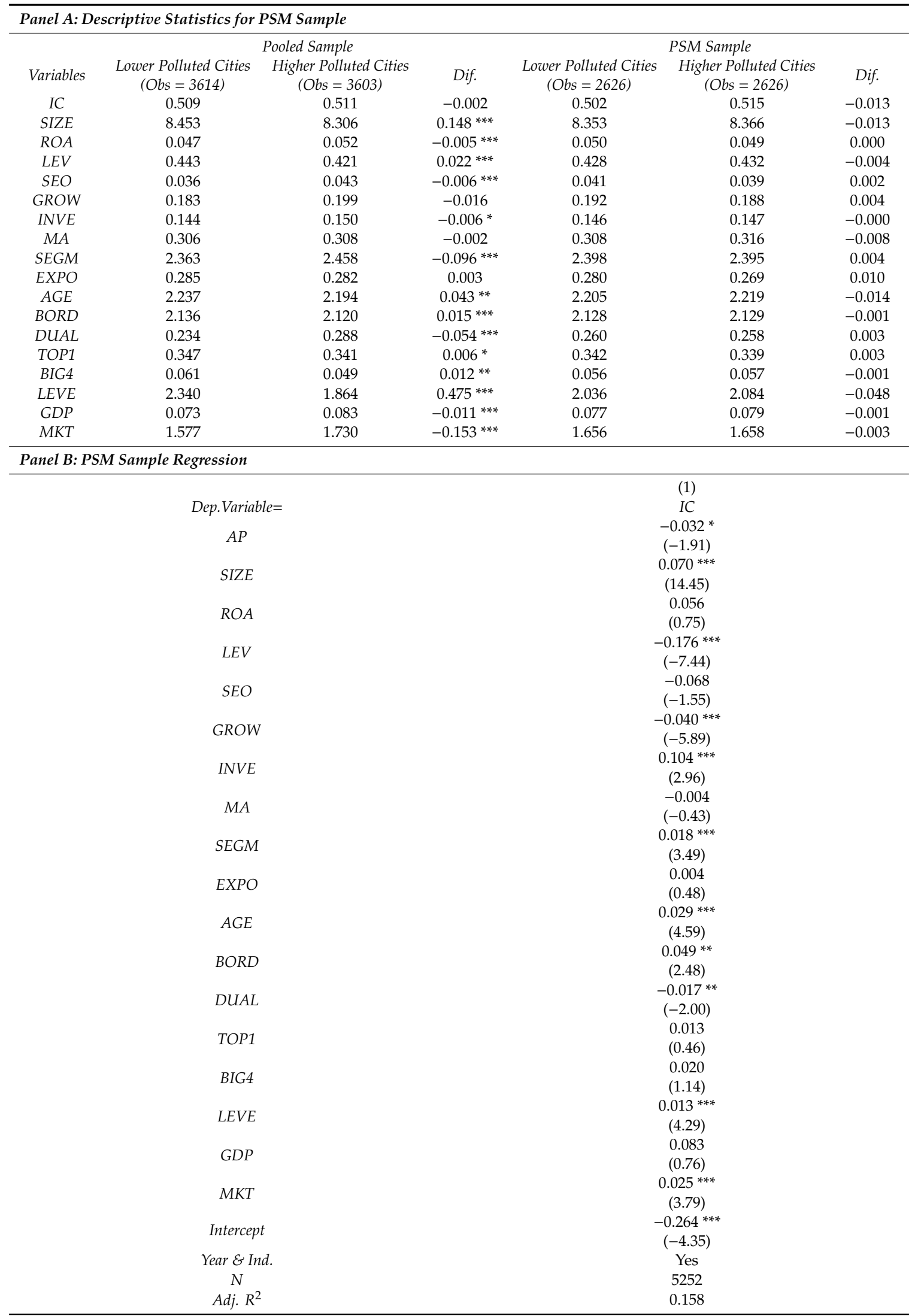

${ }^{*}, *$, and ${ }^{* * *}$ indicate statistical significance at the $10 \%, 5 \%$, and $1 \%$ levels, respectively. The $z$-values are shown in the parentheses. The standard errors are clustered at the firm level. 
Table 15. Alternative Sample and Measures.

\begin{tabular}{|c|c|c|c|c|c|c|}
\hline & (1) & (2) & (3) & (4) & (5) & (6) \\
\hline & \multicolumn{2}{|c|}{$\begin{array}{l}\text { Exclude Most Polluted } \\
\text { Provinces/Cites }\end{array}$} & \multicolumn{2}{|c|}{ Alternative $I C$ Measure } & \multicolumn{2}{|c|}{ Alternative AP Measure } \\
\hline Dep. Variable $=$ & IC & IC & IC & IC & IC & IC \\
\hline$A P$ & $\begin{array}{c}-0.062 * * * \\
(-3.04)\end{array}$ & $\begin{array}{c}-0.064^{* * *} \\
(-3.07)\end{array}$ & $\begin{array}{l}-0.110 * * \\
(-2.05)\end{array}$ & $\begin{array}{c}-0.921 * \\
(-1.77)\end{array}$ & $\begin{array}{c}-0.000 * * * \\
(-2.59)\end{array}$ & $\begin{array}{c}-0.062 * * \\
(-2.39)\end{array}$ \\
\hline SIZE & $\begin{array}{c}0.071^{* * *} \\
(13.16)\end{array}$ & $\begin{array}{c}0.072 * * * \\
(13.36)\end{array}$ & $\begin{array}{c}0.199 * * * \\
(13.36)\end{array}$ & $\begin{array}{c}1.978^{* * *} \\
(12.96)\end{array}$ & $\begin{array}{c}0.072^{* * *} \\
(13.58)\end{array}$ & $\begin{array}{l}0.072 * * * \\
(13.56)\end{array}$ \\
\hline$R O A$ & $\begin{array}{l}-0.000 \\
(-0.00)\end{array}$ & $\begin{array}{l}-0.014 \\
(-0.18)\end{array}$ & $\begin{array}{l}0.000 \\
(0.00)\end{array}$ & $\begin{array}{l}1.626 \\
(0.73)\end{array}$ & $\begin{array}{l}-0.004 \\
(-0.04)\end{array}$ & $\begin{array}{l}-0.002 \\
(-0.03)\end{array}$ \\
\hline$L E V$ & $\begin{array}{l}-0.182^{* * *} \\
(-6.96)\end{array}$ & $\begin{array}{c}-0.183^{* * *} \\
(-6.94)\end{array}$ & $\begin{array}{l}-0.526^{* * *} \\
(-7.22)\end{array}$ & $\begin{array}{l}-5.397^{* * *} \\
(-7.28)\end{array}$ & $\begin{array}{c}-0.187^{* * * *} \\
(-7.24)\end{array}$ & $\begin{array}{l}-0.187^{* * *} \\
(-7.23)\end{array}$ \\
\hline$S E O$ & $\begin{array}{l}-0.080 * * \\
(-2.00)\end{array}$ & $\begin{array}{c}-0.080 * * \\
(-2.08)\end{array}$ & $\begin{array}{l}-0.209^{*} \\
(-1.92)\end{array}$ & $\begin{array}{l}-2.704^{* *} \\
(-2.49)\end{array}$ & $\begin{array}{c}-0.082 * * \\
(-2.17)\end{array}$ & $\begin{array}{l}-0.082 * * \\
(-2.15)\end{array}$ \\
\hline GROW & $\begin{array}{l}-0.037^{* * *} \\
(-5.91)\end{array}$ & $\begin{array}{l}-0.038^{* * *} \\
(-6.12)\end{array}$ & $\begin{array}{l}-0.112 * * * \\
(-6.56)\end{array}$ & $\begin{array}{c}-1.147^{* * *} \\
(-5.95)\end{array}$ & $\begin{array}{c}-0.039^{* * *} \\
(-6.48)\end{array}$ & $\begin{array}{l}-0.039 * * * \\
(-6.47)\end{array}$ \\
\hline INVE & $\begin{array}{l}0.060 \\
(1.56)\end{array}$ & $\begin{array}{c}0.071 * \\
(1.86)\end{array}$ & $\begin{array}{c}0.208^{* *} \\
(1.97)\end{array}$ & $\begin{array}{c}2.893^{* * *} \\
(2.71)\end{array}$ & $\begin{array}{c}0.078 * * \\
(2.06)\end{array}$ & $\begin{array}{c}0.077^{* *} \\
(2.06)\end{array}$ \\
\hline$M A$ & $\begin{array}{l}-0.004 \\
(-0.50)\end{array}$ & $\begin{array}{l}-0.002 \\
(-0.25)\end{array}$ & $\begin{array}{l}-0.007 \\
(-0.36)\end{array}$ & $\begin{array}{l}-0.052 \\
(-0.25)\end{array}$ & $\begin{array}{l}-0.003 \\
(-0.45)\end{array}$ & $\begin{array}{l}-0.003 \\
(-0.46)\end{array}$ \\
\hline SEGM & $\begin{array}{c}0.017^{* * *} \\
(3.10)\end{array}$ & $\begin{array}{c}0.015^{* * *} \\
(2.78)\end{array}$ & $\begin{array}{c}0.050 * * * \\
(3.30)\end{array}$ & $\begin{array}{l}0.434^{* * *} \\
(2.99)\end{array}$ & $\begin{array}{c}0.017 \text { *** } \\
(3.23)\end{array}$ & $\begin{array}{c}0.017^{* * *} \\
(3.21)\end{array}$ \\
\hline EXPO & $\begin{array}{l}-0.000 \\
(-0.01)\end{array}$ & $\begin{array}{l}0.003 \\
(0.31)\end{array}$ & $\begin{array}{l}0.008 \\
(0.32)\end{array}$ & $\begin{array}{l}0.127 \\
(0.55)\end{array}$ & $\begin{array}{l}0.003 \\
(0.33)\end{array}$ & $\begin{array}{l}0.002 \\
(0.29)\end{array}$ \\
\hline$A G E$ & $\begin{array}{c}0.026^{* * *} \\
(3.73)\end{array}$ & $\begin{array}{c}0.026^{* * *} \\
(3.78)\end{array}$ & $\begin{array}{c}0.076^{* * *} \\
(3.93)\end{array}$ & $\begin{array}{c}0.451^{* *} \\
(2.37)\end{array}$ & $\begin{array}{c}0.027 * * * \\
(3.86)\end{array}$ & $\begin{array}{c}0.027 * * * \\
(3.86)\end{array}$ \\
\hline$B O R D$ & $\begin{array}{l}0.021 \\
(0.95)\end{array}$ & $\begin{array}{l}0.030 \\
(1.41)\end{array}$ & $\begin{array}{l}0.085 \\
(1.44)\end{array}$ & $\begin{array}{l}0.646 \\
(1.13)\end{array}$ & $\begin{array}{l}0.033 \\
(1.60)\end{array}$ & $\begin{array}{l}0.033 \\
(1.58)\end{array}$ \\
\hline DUAL & $\begin{array}{c}-0.020 * * \\
(-2.22)\end{array}$ & $\begin{array}{c}-0.021 \text { ** } \\
(-2.35)\end{array}$ & $\begin{array}{c}-0.054^{* *} \\
(-2.13)\end{array}$ & $\begin{array}{c}-0.466 \text { * } \\
(-1.93)\end{array}$ & $\begin{array}{c}-0.019 * * \\
(-2.12)\end{array}$ & $\begin{array}{c}-0.019 \text { ** } \\
(-2.11)\end{array}$ \\
\hline TOP1 & $\begin{array}{l}0.022 \\
(0.72)\end{array}$ & $\begin{array}{l}0.019 \\
(0.64)\end{array}$ & $\begin{array}{l}0.062 \\
(0.75)\end{array}$ & $\begin{array}{l}0.816 \\
(1.03)\end{array}$ & $\begin{array}{l}0.021 \\
(0.71)\end{array}$ & $\begin{array}{l}0.020 \\
(0.69)\end{array}$ \\
\hline BIG4 & $\begin{array}{l}0.027 \\
(1.34)\end{array}$ & $\begin{array}{l}0.030 \\
(1.46)\end{array}$ & $\begin{array}{l}0.075 \\
(1.32)\end{array}$ & $\begin{array}{c}1.108 \text { * } \\
(1.86)\end{array}$ & $\begin{array}{l}0.027 \\
(1.34)\end{array}$ & $\begin{array}{l}0.027 \\
(1.34)\end{array}$ \\
\hline OWNER & $\begin{array}{c}0.015^{* * *} \\
(4.26)\end{array}$ & $\begin{array}{c}0.014^{* * *} \\
(4.13)\end{array}$ & $\begin{array}{c}0.037^{* * *} \\
(3.96)\end{array}$ & $\begin{array}{c}0.389 * * * \\
(4.26)\end{array}$ & $\begin{array}{c}0.014^{* * *} \\
(4.15)\end{array}$ & $\begin{array}{c}0.014^{* * *} \\
(4.14)\end{array}$ \\
\hline GDP & $\begin{array}{l}0.071 \\
(0.62)\end{array}$ & $\begin{array}{l}0.050 \\
(0.44)\end{array}$ & $\begin{array}{l}0.182 \\
(0.59)\end{array}$ & $\begin{array}{l}0.241 \\
(0.08)\end{array}$ & $\begin{array}{l}0.065 \\
(0.60)\end{array}$ & $\begin{array}{l}0.073 \\
(0.68)\end{array}$ \\
\hline$M K T$ & $\begin{array}{c}0.031^{* * *} \\
(4.05)\end{array}$ & $\begin{array}{c}0.030^{* * *} \\
(3.93)\end{array}$ & $\begin{array}{c}0.071^{* * *} \\
(3.43)\end{array}$ & $\begin{array}{c}0.643^{* * *} \\
(3.22)\end{array}$ & $\begin{array}{c}0.025^{* * *} \\
(3.38)\end{array}$ & $\begin{array}{c}0.026^{* * *} \\
(3.48)\end{array}$ \\
\hline Intercept & $\begin{array}{c}-0.177^{* * *} \\
(-2.63)\end{array}$ & $\begin{array}{c}-0.201^{* * * *} \\
(-3.07)\end{array}$ & $\begin{array}{c}-0.744^{* * *} \\
(-4.05)\end{array}$ & $\begin{array}{c}27.277^{* * *} \\
(15.33)\end{array}$ & $\begin{array}{c}-0.260^{* * * *} \\
(-4.09)\end{array}$ & $\begin{array}{c}-0.253^{* * * *} \\
(-3.97)\end{array}$ \\
\hline Year E Ind. & Yes & Yes & Yes & Yes & Yes & Yes \\
\hline$N$ & 6638 & 6890 & 7227 & 7227 & 7227 & 7227 \\
\hline Adj. $R^{2}$ & 0.142 & 0.148 & 0.156 & 0.140 & 0.147 & 0.147 \\
\hline
\end{tabular}

$*, * *$, and ${ }^{* * *}$ indicate statistical significance at the $10 \%, 5 \%$, and $1 \%$ levels, respectively. The $t$-values are shown in parentheses. The standard errors are clustered at the firm level. 
Table 16. Control for the Climatic Condition.

\begin{tabular}{|c|c|c|c|c|}
\hline & (1) & (2) & (3) & $(4)$ \\
\hline $\begin{array}{c}\text { Dep. } \text { Variable }= \\
\text { AP }\end{array}$ & $\begin{array}{c}I C \\
-0.042 * * \\
(-2.21)\end{array}$ & $\begin{array}{c}I C \\
-\mathbf{0 . 0 3 7} * \\
\mathbf{( - 1 . 9 5 )}\end{array}$ & $\begin{array}{c}I C \\
-\mathbf{0 . 0 4 4} * * \\
\mathbf{( - 2 . 2 8 )}\end{array}$ & $\begin{array}{c}I C \\
-\mathbf{0 . 0 4 0} * * \\
\mathbf{( - 2 . 0 8 )}\end{array}$ \\
\hline HOT & $\begin{array}{l}0.298 \\
(1.11)\end{array}$ & & & \\
\hline WIND & & $\begin{array}{c}-0.060 * * * \\
(-2.75)\end{array}$ & & \\
\hline$B A D$ & & & $\begin{array}{l}-0.042 \\
(-1.05)\end{array}$ & \\
\hline CLIMATE & & & & $\begin{array}{l}0.004 \\
(1.15)\end{array}$ \\
\hline$S I Z E$ & $\begin{array}{c}0.072^{* * *} \\
(13.53)\end{array}$ & $\begin{array}{c}0.072 * * * \\
(13.53)\end{array}$ & $\begin{array}{c}0.072^{* * *} \\
(13.54)\end{array}$ & $\begin{array}{c}0.072 * * * \\
(13.53)\end{array}$ \\
\hline$R O A$ & $\begin{array}{l}-0.001 \\
(-0.01)\end{array}$ & $\begin{array}{l}0.002 \\
(0.03)\end{array}$ & $\begin{array}{l}-0.002 \\
(-0.02)\end{array}$ & $\begin{array}{l}-0.001 \\
(-0.01)\end{array}$ \\
\hline$L E V$ & $\begin{array}{c}-0.188^{* * *} \\
(-7.23)\end{array}$ & $\begin{array}{c}-0.189 * * * \\
(-7.28)\end{array}$ & $\begin{array}{c}-0.187^{* * *} \\
(-7.24)\end{array}$ & $\begin{array}{c}-0.188^{* * *} \\
(-7.23)\end{array}$ \\
\hline SEO & $\begin{array}{c}-0.081^{* *} \\
(-2.14)\end{array}$ & $\begin{array}{c}-0.080 * * \\
(-2.11)\end{array}$ & $\begin{array}{c}-0.081^{* *} \\
(-2.13)\end{array}$ & $\begin{array}{c}-0.082 \text { ** } \\
(-2.15)\end{array}$ \\
\hline GROW & $\begin{array}{c}-0.039 * * * \\
(-6.46)\end{array}$ & $\begin{array}{c}-0.039 * * * \\
(-6.48)\end{array}$ & $\begin{array}{c}-0.039 * * * \\
(-6.48)\end{array}$ & $\begin{array}{c}-0.039 * * * \\
(-6.46)\end{array}$ \\
\hline$I N V E$ & $\begin{array}{c}0.076^{* *} \\
(2.04)\end{array}$ & $\begin{array}{c}0.079 * * \\
(2.12)\end{array}$ & $\begin{array}{c}0.079 * * \\
(2.09)\end{array}$ & $\begin{array}{c}0.077^{* *} \\
(2.04)\end{array}$ \\
\hline$M A$ & $\begin{array}{l}-0.003 \\
(-0.46)\end{array}$ & $\begin{array}{l}-0.003 \\
(-0.42)\end{array}$ & $\begin{array}{l}-0.003 \\
(-0.44)\end{array}$ & $\begin{array}{l}-0.003 \\
(-0.46)\end{array}$ \\
\hline SEGM & $\begin{array}{c}0.018^{* * *} \\
(3.26)\end{array}$ & $\begin{array}{c}0.017^{* * *} \\
(3.17)\end{array}$ & $\begin{array}{l}0.018^{* * *} \\
(3.23)\end{array}$ & $\begin{array}{c}0.018^{* * *} \\
(3.24)\end{array}$ \\
\hline EXPO & $\begin{array}{l}0.002 \\
(0.25)\end{array}$ & $\begin{array}{l}0.002 \\
(0.28)\end{array}$ & $\begin{array}{l}0.003 \\
(0.32)\end{array}$ & $\begin{array}{l}0.002 \\
(0.25)\end{array}$ \\
\hline$A G E$ & $\begin{array}{c}0.026^{* * * *} \\
(3.82)\end{array}$ & $\begin{array}{c}0.027^{* * * *} \\
(3.93)\end{array}$ & $\begin{array}{c}0.026^{* * *} \\
(3.84)\end{array}$ & $\begin{array}{c}0.027^{* * * *} \\
(3.86)\end{array}$ \\
\hline$B O R D$ & $\begin{array}{l}0.033 \\
(1.58)\end{array}$ & $\begin{array}{l}0.033 \\
(1.59)\end{array}$ & $\begin{array}{l}0.033 \\
(1.58)\end{array}$ & $\begin{array}{l}0.033 \\
(1.59)\end{array}$ \\
\hline DUAL & $\begin{array}{c}-0.019^{* *} \\
(-2.09)\end{array}$ & $\begin{array}{c}-0.019 * * \\
(-2.09)\end{array}$ & $\begin{array}{c}-0.019 * * \\
(-2.12)\end{array}$ & $\begin{array}{c}-0.019 * * \\
(-2.09)\end{array}$ \\
\hline TOP1 & $\begin{array}{l}0.020 \\
(0.69)\end{array}$ & $\begin{array}{l}0.023 \\
(0.76)\end{array}$ & $\begin{array}{l}0.021 \\
(0.70)\end{array}$ & $\begin{array}{l}0.021 \\
(0.71)\end{array}$ \\
\hline BIG4 & $\begin{array}{l}0.027 \\
(1.34)\end{array}$ & $\begin{array}{l}0.027 \\
(1.34)\end{array}$ & $\begin{array}{l}0.027 \\
(1.33)\end{array}$ & $\begin{array}{l}0.027 \\
(1.34)\end{array}$ \\
\hline LEVE & $\begin{array}{c}0.014^{* * *} \\
(4.14)\end{array}$ & $\begin{array}{c}0.014^{* * *} \\
(4.11)\end{array}$ & $\begin{array}{c}0.014^{* * *} \\
(4.12)\end{array}$ & $\begin{array}{c}0.014^{* * *} \\
(4.13)\end{array}$ \\
\hline GDP & $\begin{array}{l}0.052 \\
(0.48)\end{array}$ & $\begin{array}{l}-0.054 \\
(-0.48)\end{array}$ & $\begin{array}{l}0.116 \\
(1.02)\end{array}$ & $\begin{array}{l}0.016 \\
(0.14)\end{array}$ \\
\hline OWNER & $\begin{array}{c}0.024^{* * *} \\
(3.24)\end{array}$ & $\begin{array}{c}0.025^{* * *} \\
(3.37)\end{array}$ & $\begin{array}{c}0.027^{* * *} \\
(3.58)\end{array}$ & $\begin{array}{c}0.024^{* * *} \\
(3.21)\end{array}$ \\
\hline Intercept & $\begin{array}{c}-0.230 * * * \\
(-3.54)\end{array}$ & $\begin{array}{c}-0.208^{* * *} \\
(-3.15)\end{array}$ & $\begin{array}{c}-0.217^{* * *} \\
(-3.25)\end{array}$ & $\begin{array}{c}-0.224^{* * *} \\
(-3.42)\end{array}$ \\
\hline Year E Ind. & Yes & Yes & Yes & Yes \\
\hline$N$ & 7227 & 7227 & 7227 & 7227 \\
\hline Adj. $R^{2}$ & 0.147 & 0.148 & 0.147 & 0.147 \\
\hline
\end{tabular}

$*, * *$, and ${ }^{* * *}$ indicate statistical significance at the $10 \%, 5 \%$, and $1 \%$ levels, respectively. The $t$-values are shown in parentheses. The standard errors are clustered at the firm level.

\section{Conclusions}

Without a thorough understanding of air pollution, its hazards and its influencing mechanisms, the process of alleviating air pollution is sure to be slow, and so may be the pace of China's high-quality 
economic development. Unlike research on the various economic, social, and medical costs of air pollution, this paper investigates the micro-institutional costs from the perspective of internal control. Using data on air pollution from cities in China and a large sample of non-financial public firms listed on the Shanghai and Shenzhen Stock Exchange from 2014 to 2016, we find that internal control quality is significantly and negatively associated with the severity of air pollution in the firm's home city. We also find that among air pollutants, $\mathrm{PM} 2.5$ and $\mathrm{SO}_{2}$ have the most significant effects, which confirms that air pollution has incurred micro-institutional costs. We also find that this effect varies in different contexts, depending on factors related to a firm's external environment, ownership features, demographic traits of the chairman, and employees. Our further analysis indicated that air pollution can result in a lower quality of accounting information, serious agency problems, and lower firm value. Besides its contribution to the literature, this study also has practical implications. First, the findings of this study confirm the importance of both China's Blue-Sky Protection Campaign and of the country moving toward an economic mode based on high-quality development. These inspiring developments should encourage all members of society to support and participate in a green, prosperous, democratic, civilized, and harmonious new China. Second, this study suggests that as one of the producers of air pollution, firms cannot protect themselves from its hazards. Therefore, firms should join in environmental governance. Finally, the government needs to enhance measures designed to protect the environment, strengthen its supervision, and enforce related laws, thereby promoting China's future development.

Author Contributions: Conceptualization, S.L. and D.Y.; methodology, S.L.; data curation, S.L.; writing—original draft preparation, X.L.; writing—-review and editing, N.L.

Funding: This research was funded by the National Natural Science Foundation of China, grant number 71790604, 71932003, 71702030, 71902030, 71902033, 71902026 and the Fundamental Research Funds for the Central Universities in UIBE, grant number 19QD08.

Conflicts of Interest: The authors declare no conflict of interest.

\section{References}

1. Zhang, I.X. Economic consequences of the Sarbanes-Oxley Act of 2002. J. Account. Econ. 2007, 44, 74-115. [CrossRef]

2. Nee, V. New Institutionalism in Economic and Sociology. In The Handbook of Economic Sociology, 2nd ed.; Neil, J.S., Richard, S., Eds.; Russell Sage: New York, NY, USA, 2005.

3. Beatty, T.K.; Shimshack, J.P. Air Pollution and Children's Respiratory Health: A Cohort Analysis. J. Environ. Econ. Manag. 2014, 67, 39-57. [CrossRef]

4. Currie, J.; Neidell, M. Air Pollution and Infant Health: What Can We Learn from California's Recent Experience? Q. J. Econ. 2005, 120, 1003-1030.

5. Power, M.C.; Kioumourtzoglou, M.-A.; Hart, J.E.; Okereke, O.I.; Laden, F.; Weisskopf, M.G. The relation between past exposure to fine particulate air pollution and prevalent anxiety: Observational cohort study. BMJ 2015, 350, 1111. [CrossRef] [PubMed]

6. Szyszkowicz, M. Air Pollution and Emergency Department Visits for Depression in Edmonton, Canada. Int. J. Occup. Med. Environ. Health 2007, 20, 241-245. [CrossRef] [PubMed]

7. Lu, J.G.; Lee, J.J.; Gino, F.; Galinsky, A.D. Polluted Morality: Air Pollution Predicts Criminal Activity and Unethical Behavior. Psychol. Sci. 2018, 29, 340-355. [CrossRef] [PubMed]

8. Chang, T.Y.; Zivin, J.G.; Gross, T.; Neidell, M. Particulate Pollution and the Productivity of Pear Packers. Am. Econ. J. Econ. Policy 2016, 8, 141-169. [CrossRef]

9. Chang, T.Y.; Zivin, J.G.; Gross, T.; Neidell, M. The Effect of Pollution on Worker Productivity: Evidence from Call-Center Workers in China. NBER Working Paper No. 22328. Am. Econ. J. Appl. Econ. 2019, 11, 151-172. [CrossRef]

10. Chang, T.Y.; Huang, W.; Wang, Y. Something in the Air: Pollution and the Demand for Health Insurance. Rev. Econ. Stud. 2018, 85, 1609-1634. [CrossRef]

11. Hanna, R.; Oliva, P. The effect of pollution on labor supply: Evidence from a natural experiment in Mexico City. J. Public Econ. 2015, 122, 68-79. [CrossRef] 
12. Luo, J. How does smog affect firms' investment behavior? A natural experiment based on a sudden surge in the PM2.5 index. China J. Account. Res. 2017, 10, 359-378. [CrossRef]

13. Zivin, J.G.; Neidell, M. The Impact of Pollution on Worker Productivity. Am. Econ. Rev. 2012, 102, 3652-3673. [CrossRef]

14. The Committee of Sponsoring Organizations (COSO). Enterprise Risk Management-Integrating with Strategy and Performance (2017). 2017. Available online: https://www.coso.org/Pages/erm.aspx (accessed on 7 September 2019).

15. Ministry of Finance of the People's Republic of China (MOF). Enterprise Internal Control Basic Norms. 2008. Available online: http://kjs.mof.gov.cn/zhuantilanmu/neibukongzhibiaozhunjianshe/200807/t20080704_55983. html (accessed on 7 September 2019).

16. Doyle, J.T.; Ge, W.; Mcvay, S. Accruals Quality and Internal Control Over Financial Reporting. Account. Rev. 2007, 82, 1141-1170. [CrossRef]

17. Ashbaugh-Skaife, H.; Collins, D.W.; Kinney, W.R.; Lafond, R. The Effect of Sox Internal Control Deficiencies and their Remediation on Accrual Quality. Account. Rev. 2008, 83, 217-250. [CrossRef]

18. Dhaliwal, D.; Hogan, C.; Trezevant, R.; Wilkins, M. Internal Control Disclosures, Monitoring, and the Cost of Debt. Account. Rev. 2011, 86, 1131-1156. [CrossRef]

19. Kim, J.B.; Song, B.Y.; Zhang, L. Internal Control Weakness and Bank Loan Contracting: Evidence from SOX Section 404 Disclosures. Account. Rev. 2011, 86, 1157-1188. [CrossRef]

20. Cheng, M.; Dhaliwal, D.S.; Zhang, Y. Does Investment Efficiency Improve after the Disclosure of Material Weaknesses in Internal Control over Financial Reporting? SSRN Electron. J. 2013, 56, 1-18.

21. Feng, M.; Li, C.; McVay, S.E.; Skaife, H.A. Does Ineffective Internal Control over Financial Reporting affect a Firm's Operations? Evidence from Firms' Inventory Management. Account. Rev. 2015, 90, 529-557. [CrossRef]

22. Chhaochharia, V.; Grinstein, Y. Corporate Governance and Firm Value: The Impact of the 2002 Governance Rules. J. Financ. 2007, 62, 1789-1825. [CrossRef]

23. Balsam, S.; Jiang, W.; Lu, B. Equity Incentives and Internal Control Weaknesses. Contemp. Account. Res. 2014, 31, 178-201. [CrossRef]

24. Goh, B.W. Audit Committees, Boards of Directors, and Remediation of Material Weaknesses in Internal Control. Contemp. Account. Res. 2009, 26, 549-579. [CrossRef]

25. Johnstone, K.; Li, C.; Rupley, K.H. Changes in Corporate Governance Associated with the Revelation of Internal Control Material Weaknesses and their Subsequent Remediation. Contemp. Account. Res. 2011, 28, 331-383. [CrossRef]

26. Krishnan, J. Audit Committee Quality and Internal Control: An Empirical Analysis. Account. Rev. 2005, 80, 649-675. [CrossRef]

27. Guo, J.; Huang, P.; Zhang, Y.; Zhou, N. The Effect of Employee Treatment Policies on Internal Control Weaknesses and Financial Restatements. Account. Rev. 2016, 91, 1167-1194. [CrossRef]

28. Kanagaretnam, K.; Lobo, G.J.; Ma, C.; Zhou, J. National Culture and Internal Control Material Weaknesses around the World. J. Account. Audit. Financ. 2016, 31, 28-50. [CrossRef]

29. Schlenker, W.; Walker, W.R. Airports, Air Pollution, and Contemporaneous Health. Rev. Econ. Stud. 2016, 83, 768-809. [CrossRef]

30. Chay, K.Y.; Greenstone, M. The Impact of Air Pollution on Infant Mortality: Evidence from Geographic Variation in Pollution Shocks Induced by a Recession. Q. J. Econ. 2003, 118, 1121-1167. [CrossRef]

31. World Health Organization (WHO). Ambient Air Pollution: A Global Assessment of Exposure and Burden of Disease. 2016. Available online: http://apps.who.int/iris/bitstream/10665/250141/1/9789241511353-eng. pdf?ua=1 (accessed on 7 September 2019).

32. World Health Organization (WHO). 2017 Global Platform on Air Quality and Health. Available online: http://www.who.int/phe/health_topics/outdoorair/global_platform/en/Yang (accessed on 18 August 2017).

33. Jazani, R.K.; Saremi, M.; Rezapour, T.; Kavousi, A.; Shirzad, H. Influence of traffic-related noise and air pollution on self-reported fatigue. Int. J. Occup. Saf. Ergon. 2015, 21, 193-200. [CrossRef] [PubMed]

34. Yang, A.C.; Tsai, A.C.S.J.; Huang, N.E. Decomposing the Association of Completed Suicide with Air Pollution, Weather, and Unemployment Data at Different Time Scales. J. Affect. Disord. 2011, 129, 275-281. [CrossRef]

35. Cheung, C.; Law, R. The impact of air quality on tourism: The case of Hong Kong. Pac. Tour. Rev. 2001, 5, 69-74. 
36. Herrnstadt, E.; Heyes, A.; Muehlegger, E.; Saberian, S. Air Pollution as A Cause of Violent Crime: Evidence from Los Angeles and Chicago. 2016. Available online: http:/www.evanherrnstadt.com/docs/ CrimePollution_JM.pdf (accessed on 7 September 2019).

37. Petrovits, C.; Shakespeare, C.; Shih, A. The Causes and Consequences of Internal Control Problems in Nonprofit Organizations. Account. Rev. 2011, 86, 325-357. [CrossRef]

38. Gong, G.; Ke, B.; Yu, Y. Home Country Investor Protection, Ownership Structure and Cross-Listed Firms' Compliance with SOX-Mandated Internal Control Deficiency Disclosures. Contemp. Account. Res. 2013, 30, 1490-1523. [CrossRef]

39. Hoitash, U.; Hoitash, R.; Bedard, J.C. Corporate Governance and Internal Control over Financial Reporting: A Comparison of Regulatory Regimes. Account. Rev. 2009, 84, 839-867. [CrossRef]

40. Li, C.; Sun, L.; Ettredge, M. Financial executive qualifications, financial executive turnover, and adverse SOX 404 opinions. J. Account. Econ. 2010, 50, 93-110. [CrossRef]

41. Yun, J. External regulation and the quality of internal control: An empirical analysis of China's A-share markets. In Proceedings of the 2016 13th International Conference on Service Systems and Service Management (ICSSSM), Kunming, China, 24-26 June 2016; pp. 1-4.

42. Murphy, S.R.; Schelegle, E.S.; Miller, L.A.; Hyde, D.M.; Van Winkle, L.S. Ozone Exposure Alters Serotonin and Serotonin Receptor Expression in the Developing Lung. Toxicol. Sci. 2013, 134, 168-179. [CrossRef] [PubMed]

43. Uboh, F. Effect of Inhalation Exposure to Gasoline on Sex Hormones Profile in Wistar Albino Rats. Acta Endocrinol. 2007, 3, 23-30. [CrossRef]

44. Pérez-González, F; Yun, H. Risk Management and Firm Value: Evidence from Weather Derivatives. J. Financ. 2013, 68, 2143-2176. [CrossRef]

45. Chen, H.; Dong, W.; Han, H.; Zhou, N. A comprehensive and quantitative internal control index: Construction, validation, and impact. Rev. Quant. Financ. Account. 2017, 49, 337-377. [CrossRef]

46. The Committee of Sponsoring Organizations (COSO). Internal Control—Integrated Framework. 1992. Available online: https://www.coso.org/Pages/guidance.aspx (accessed on 7 September 2019).

47. Wang, X.L.; Fan, G.; Hu, L.P. Marketization Index of China's Provinces: Neri Report; Social Sciences Academic Press: Beijing, China, 2016.

48. Chen, H.; Yang, D.; Zhang, X.; Zhou, N. The Moderating Role of Internal Control in Tax Avoidance: Evidence from a COSO-Based Internal Control Index in China. J. Am. Tax. Assoc. 2019. [CrossRef]

49. Chen, S.; Sun, Z.; Tang, S.; Wu, D. Government intervention and investment efficiency: Evidence from China. J. Corp. Financ. 2011, 17, 259-271. [CrossRef]

50. Fama, E.F.; Jensen, M.C. Separation of Ownership and Control. J. Law Econ. 1983, 26, 301-325. [CrossRef]

51. Bloom, N.; Bond, S.; van Reenen, J. Uncertainty and Investment Dynamics. Rev. Econ. Stud. 2007, 74, $391-415$. [CrossRef]

52. Chay, J.; Suh, J. Payout policy and cash-flow uncertainty. J. Financ. Econ. 2009, 93, 88-107. [CrossRef]

53. Covas, F.; den Haan, W.J. The Cyclical Behavior of Debt and Equity Finance. Am. Econ. Rev. 2017, 101, 877-899. [CrossRef]

54. Hasan, M.; Taylor, G.; Richardson, G. Does a firm's life cycle explain its propensity to engage in corporate tax avoidance? Eur. Account. Rev. 2017, 26, 469-501. [CrossRef]

55. Chhaochharia, V.; Grinstein, Y.; Grullon, G.; Michaely, R. Product market competition and internal governance: Evidence from the Sarbanes-Oxley Act. Manag. Sci. 2016, 63, 1405-1424. [CrossRef]

56. Giroud, X.; Mueller, H.M. Corporate Governance, Product Market Competition, and Equity Prices. J. Financ. 2011, 66, 563-600. [CrossRef]

57. Zhang, C.; Chen, H. Product market competition, state ownership and internal control quality. China J. Account. Stud. 2016, 4, 1-27. [CrossRef]

58. Gaspar, J.M.; Massa, M.; Matos, P. Favoritism in Mutual Fund Families? Evidence on Strategic Cross-Fund Subsidization. J. Financ. 2006, 61, 73-104. [CrossRef]

59. Peress, J. Product Market Competition, Insider Trading, and Stock Market Efficiency. J. Financ. 2010, 65, 1-43. [CrossRef]

60. Huang, J.; Kisgen, D.J. Gender and corporate finance: Are male executives overconfident relative to female executives? J. Financ. Econ. 2013, 108, 822-839. [CrossRef] 
61. Srinidhi, B.; Gul, F.A.; Tsui, J. Female Directors and Earnings Quality. Contemp. Account. Res. 2011, 28, 1610-1644. [CrossRef]

62. Fraser, S.; Greene, F.J. The Effects of Experience on Entrepreneurial Optimism and Uncertainty. Economica 2006, 73, 169-192. [CrossRef]

63. Ge, W.; Li, Z.; Liu, Q.; McVay, S. When Does Internal Control over Financial Reporting Curb Resource Extraction? Evidence from China; Working Paper; University of Washington: Washington, DC, USA, 2019.

64. Goh, B.W.; Li, D. Internal Controls and Conditional Conservatism. Account. Rev. 2011, 86, 975-1005. [CrossRef]

65. Khan, M.; Watts, R.L. Estimation and Empirical Properties of a Firm-Year Measure of Accounting Conservatism. J. Account. Econ. 2009, 48, 132-150. [CrossRef]

66. Chen, Y.; Ebenstein, A.; Greenstone, M.; Li, H. Evidence on the impact of sustained exposure to air pollution on life expectancy from China's Huai River policy. Proc. Natl. Acad. Sci. USA 2013, 110, 12936-12941. [CrossRef] [PubMed]

67. Ebenstein, A.; Fan, M.; Greenstone, M.; He, G.; Zhou, M. New evidence on the impact of sustained exposure to air pollution on life expectancy from China's Huai River Policy. Proc. Natl. Acad. Sci. USA 2017, 114, 10384-10389. [CrossRef] [PubMed]

68. Madsen, J.; DeHaan, E.; Piotroski, J.D. Do Weather-Induced Moods Affect the Processing of Earnings News? J. Account. Res. 2017, 75, 245-550.

69. Hirshleifer, D.; Shumway, T. Good Day Sunshine: Stock Returns and the Weather. J. Financ. 2003, 58, 1009-1032. [CrossRef]

(C) 2019 by the authors. Licensee MDPI, Basel, Switzerland. This article is an open access article distributed under the terms and conditions of the Creative Commons Attribution (CC BY) license (http://creativecommons.org/licenses/by/4.0/). 